TRANSACTIONS OF THE

AMERICAN MATHEMATICAL SOCIETY

Volume 358, Number 11, November 2006, Pages 5025-5057

S 0002-9947(06)03931-6

Article electronically published on June 13, 2006

\title{
INTRINSIC ULTRACONTRACTIVITY OF THE FEYNMAN-KAC SEMIGROUP FOR RELATIVISTIC STABLE PROCESSES
}

\author{
TADEUSZ KULCZYCKI AND BARTŁOMIEJ SIUDEJA
}

\begin{abstract}
Let $X_{t}$ be the relativistic $\alpha$-stable process in $\mathbf{R}^{d}, \alpha \in(0,2), d>\alpha$, with infinitesimal generator $H_{0}^{(\alpha)}=-\left(\left(-\Delta+m^{2 / \alpha}\right)^{\alpha / 2}-m\right)$. We study intrinsic ultracontractivity (IU) for the Feynman-Kac semigroup $T_{t}$ for this process with generator $H_{0}^{(\alpha)}-V, V \geq 0, V$ locally bounded. We prove that if $\lim _{|x| \rightarrow \infty} V(x)=\infty$, then for every $t>0$ the operator $T_{t}$ is compact. We consider the class $\mathcal{V}$ of potentials $V$ such that $V \geq 0, \lim _{|x| \rightarrow \infty} V(x)=\infty$ and $V$ is comparable to the function which is radial, radially nondecreasing and comparable on unit balls. For $V$ in the class $\mathcal{V}$ we show that the semigroup $T_{t}$ is IU if and only if $\lim _{|x| \rightarrow \infty} V(x) /|x|=\infty$. If this condition is satisfied we also obtain sharp estimates of the first eigenfunction $\phi_{1}$ for $T_{t}$. In particular, when $V(x)=|x|^{\beta}, \beta>0$, then the semigroup $T_{t}$ is IU if and only if $\beta>1$. For $\beta>1$ the first eigenfunction $\phi_{1}(x)$ is comparable to
\end{abstract}

$$
\exp \left(-m^{1 / \alpha}|x|\right)(|x|+1)^{(-d-\alpha-2 \beta-1) / 2} .
$$

\section{INTRODUCTION}

The purpose of this paper is to study the Feynman-Kac semigroup for the relativistic $\alpha$-stable process $X_{t}$ on $\mathbf{R}^{d}, \alpha \in(0,2)$. This process is a Markov process with independent and homogeneous increments and characteristic function of the form

$$
\mathbf{E}^{0}\left(\exp \left(i \xi X_{t}\right)\right)=\exp \left(-t\left(\left(m^{2 / \alpha}+|\xi|^{2}\right)^{\alpha / 2}-m\right)\right)
$$

where $\xi \in \mathbf{R}^{d}, m>0, t>0$. In the entire paper we assume that $d>\alpha$. As usual $\mathbf{E}^{x}, x \in \mathbf{R}^{d}$, denotes the expected value for the process starting from $x \in \mathbf{R}^{d}$.

The Feynman-Kac semigroup $T_{t}, t>0$, for $X_{t}$ and measurable, locally bounded potential $0 \leq V(x)<\infty$ is defined as follows:

$$
T_{t}(f)(x)=\mathbf{E}^{x}\left(\exp \left(-\int_{0}^{t} V\left(X_{s}\right) d s\right) f\left(X_{t}\right)\right), x \in \mathbf{R}^{d}, f \in L^{2}\left(\mathbf{R}^{d}\right) .
$$

The generator of this semigroup is the Schrödinger operator $H^{(\alpha)}=H_{0}^{(\alpha)}-V$, where

$$
H_{0}^{(\alpha)}=-\left(\left(-\Delta+m^{2 / \alpha}\right)^{\alpha / 2}-m\right)
$$

Received by the editors March 23, 2004 and, in revised form, November 11, 2004.

2000 Mathematics Subject Classification. Primary 47G30, 60G51.

Key words and phrases. Intrinsic ultracontractivity, relativistic, Feynman-Kac semigroup, Schrödinger operator, first eigenfunction.

This work was supported by KBN grant 2 P03A 04122 and RTN Harmonic Analysis and Related Problems, contract HPRN-CT-2001-00273-HARP. 
In this paper we study the Feynman-Kac semigroup for the generator $H^{(\alpha)}=$ $H_{0}^{(\alpha)}-V$ by using methods of stochastic processes. Although proofs are rather complicated they are quite general and can be applied to many other operators e.g. $-(-\Delta)^{\alpha / 2}$, which are generators of symmetric $\alpha$-stable processes. It is worth pointing out that Feynman-Kac semigroups for Markov processes (especially for symmetric $\alpha$-stable processes) have been widely studied ([Z], BB1, BB2, [CS1, CS2]).

The relativistic $\alpha$-stable process has been introduced and studied in $[\mathrm{R}]$. For $\alpha=1$ this process has been studied in [CMS] (see also [C1], Ba1] and [Ba2]). For $\alpha=1$ the generator of this process has the form

$$
H_{0}^{(1)}=-\left(\sqrt{-\Delta+m^{2}}-m\right)
$$

and $-H_{0}^{(1)}$ is called relativistic Hamiltonian. As explained in CMS this operator corresponds to the kinetic energy of a relativistic particle with mass $m$. If $p$ is the momentum of the particle, then its relativistic kinetic energy is given by $E=$ $\sqrt{p^{2}+m^{2}}$. In the process of quantization the momentum $p$ is replaced by the differential operator $-i \nabla$, and the quantum analog of the relativistic kinetic energy is the free relativistic Hamiltonian $-H_{0}^{(1)}$.

There are many problems in quantum mechanics which can be formulated in terms of such generators. For example they were investigated by E. Lieb in difficult problems concerning the stability of relativistic matter. There exists an important literature on properties of relativistic Hamiltonians $(\overline{\mathrm{L}}, \overline{\mathrm{H}}, \overline{\mathrm{DL}}, \overline{\mathrm{Db}}, \mathrm{F}, \mathrm{FL}$, $[\mathrm{LY}]$ ).

Now we come back to formulating results for the Feynman-Kac semigroup $T_{t}$ of the relativistic $\alpha$-stable process. Let us recall that we assume in this paper that the potential $V$ which appears in the definition of the Feynman-Kac semigroup satisfies $0 \leq V(x)<\infty$. The Feynman-Kac semigroup $T_{t}$ is given by the kernel $u(t, x, y)$, that is,

$$
T_{t} f(x)=\int_{\mathbf{R}^{d}} u(t, x, y) f(y) d y, x \in \mathbf{R}^{d}, f \in L^{2}\left(\mathbf{R}^{d}\right) .
$$

For each $t>0$ the kernel $u(t, x, y)$ is continuous and bounded on $\mathbf{R}^{d} \times \mathbf{R}^{d}$. For any $t>0, x, y \in \mathbf{R}^{d}$ the kernel is strictly positive. The proof of these properties is standard. It is similar to proofs for the classical Feynman-Kac semigroup (see e.g. CZ]. For the convienience of the reader we write the short proof of properties of $u(t, x, y)$ in Lemma 3.1 .

Our first result gives an easy criterion for compactness of operators $T_{t}$.

Theorem 1.1. If $V(x) \longrightarrow \infty$ as $|x| \longrightarrow \infty$, then for all $t>0$ the operators $T_{t}$ are compact. If there exists a set consisting of an infinite number of disjoint unit balls such that $V(x)$ is bounded on this set, then for all $t>0$ the operators $T_{t}$ are not compact.

From now on we will assume that $V(x) \longrightarrow \infty$ as $|x| \longrightarrow \infty$. The properties of $u(t, x, y)$ and general theory of semigroups for compact operators gives the following standard results. There exists an orthonormal basis in $L^{2}\left(\mathbf{R}^{d}\right)$ of eigenfunctions $\left\{\phi_{n}\right\}_{n=1}^{\infty}$ with corresponding eigenvalues $\left\{e^{-\lambda_{n} t}\right\}_{n=1}^{\infty}$ satisfying $0<\lambda_{1}<\lambda_{2} \leq \lambda_{3} \leq$ $\cdots$ and $\lim _{n \rightarrow \infty} \lambda_{n}=\infty$. That is, $T_{t} \phi_{n}=e^{-\lambda_{n} t} \phi_{n}$. All $\phi_{n}$ are continuous and bounded. The first eigenfunction $\phi_{1}$ is strictly positive. 
The most important result of this paper concerns intrinsic ultracontractivity (IU) for the semigroup $T_{t}$. IU was introduced by E. B. Davies and B. Simon in [DS]. The semigroup $T_{t}$ is called intrinsically ultracontractive if and only if for any $t>0$ there exists a constant $C_{t}$ such that for all $x, y \in \mathbf{R}^{d}$

$$
u(t, x, y) \leq C_{t} \phi_{1}(x) \phi_{1}(y) .
$$

This definition comes from [DS], Theorem 3.2(iv), which presents many equivalent conditions for IU. It is well known that the upper bound inequality implies the lower bound inequality (see [DS], Theorem 3.2, proof of (iv) $\Rightarrow(\mathrm{v})$ ). Therefore IU may also be formulated in the following way. The semigroup $T_{t}$ is called intrinsically ultracontractive if and only if for any $t>0$ there exist constants $C_{t}, c_{t}>0$ such that for all $x, y \in \mathbf{R}^{d}$

$$
c_{t} \phi_{1}(x) \phi_{1}(y) \leq u(t, x, y) \leq C_{t} \phi_{1}(x) \phi_{1}(y) .
$$

There are many other equivalent conditions for IU (see [DS, B] ).

Let us point out that in [DS] it is assumed that $\int_{\mathbf{R}^{d}} u(t, x, x) d x<\infty$, and we do not assume this apriori. Nevertheless we do not use this assumption anywhere, and also the proof of (1.2) $\Rightarrow(1.3)$ does not use this assumption.

In this paper we will check IU using the following conditions which may be studied using probabilistic methods.

Condition 1.2. There exists an open, bounded and nonempty set $D$ such that for any $t>0$ there is a constant $c_{t, D}>0$ such that for any $x \in \mathbf{R}^{d}$,

$$
T_{t}\left(1_{\mathbf{R}^{d}}\right)(x) \leq c_{t, D} T_{t}\left(1_{D}\right)(x) .
$$

Condition 1.3. For any open, bounded and nonempty set $D$ and for any $t>0$ there is a constant $c_{t, D}>0$ such that for any $x \in \mathbf{R}^{d}$,

$$
T_{t}\left(1_{B(x, 1)}\right)(x) \leq c_{t, D} T_{t}\left(1_{D}\right)(x) .
$$

Condition 1.2 implies IU. We will show this at the end of Section 3. The fact that Condition 1.2 implies IU is rather well known (see e.g. [BD], Lemma 1.4). However we could not find in the literature the direct proof for the Feynman-Kac semigroup. Therefore we decided to provide the brief proof.

Also at the end of Section 3 we will show that IU implies Condition 1.3. This condition will be used to show that for some potential $V$ the semigroup is not IU.

IU has been introduced in DS for very general semigroups. Important examples of such semigroups are the semigroups of elliptic operators $H_{0}$ and the semigroups for Schrödinger operators $H=H_{0}-V$ both on $\mathbf{R}^{d}$, as well as on domains $D$ (with Dirichlet boundary conditions). IU for such semigroups has been widely studied (see e.g. [B], Da $[\mathrm{D}, \mathrm{BD}$ ). IU has also been studied for semigroups generated by $-(-\Delta)^{\alpha / 2}$ (see e.g. [K], CS1], CS2]).

The classical result for Feynman-Kac semigroups $T_{t}$ on $\mathbf{R}^{d}$ generated by $H=$ $\Delta-V$ is the following fact (Corollary 4.5.5, Theorem 4.5.11 and Corollary 4.5.8 in (D)). If $V(x)=|x|^{\beta}$ (dimension $d \geq 1$ ), then $T_{t}$ is IU iff $\beta>2$. Moreover for $\beta>2$ we have

$$
c f(x) \leq \phi_{1}(x) \leq C f(x),|x|>1,
$$

where $c$ and $C$ are positive constants, and

$$
f(x)=|x|^{-\beta / 4+(d-1) / 2} \exp \left(-\frac{2}{2+\beta}|x|^{1+\beta / 2}\right) .
$$


There are of course many other results of similar type (see e.g. Theorems 6.1,6.3, 6.4 in [DS]).

Now we will define the class $\mathcal{V}$ of potentials which we will investigate in this paper. First we need the definition of the auxiliary class of functions $\mathcal{L}$.

Definition 1.4. We say that a function $L:[0, \infty) \longrightarrow[0, \infty)$ belongs to the class $\mathcal{L}$ if

(1) $L$ is nondecreasing,

(2) $\lim _{t \rightarrow \infty} L(t)=\infty$,

(3) there exists $\tilde{c} \geq 1$ such that for all $t \geq 0$

$$
L(t+1) \leq \tilde{c} L(t)+\tilde{c} .
$$

Definition 1.5. We say that the potential $V: \mathbf{R}^{d} \longrightarrow[0, \infty)$ belongs to the class $\mathcal{V}$ if there exists a function $L \in \mathcal{L}$ and a constant $C>0$ such that for any $x \in \mathbf{R}^{d}$ we have

$$
L(|x|) \leq V(x) \leq C L(|x|)+C .
$$

Roughly speaking $V \in \mathcal{V}$ if $V \geq 0, \lim _{|x| \rightarrow \infty} V(x)=\infty$ and $V$ is comparable to the function which is radial, radially nondecreasing and comparable on unit balls. Typical examples of functions belonging to $\mathcal{V}$ are $V(x)=|x|^{\beta}$ for $\beta>0$, $V(x)=|x|^{\beta} \ln ^{\gamma}(|x|+2)$ for $\beta \geq 0$ and $\gamma>0$, and $V(x)=e^{\beta|x|}$ for $\beta>0$. On the other hand $V(x)=\exp \left(|x|^{\beta}\right), \beta>1$, does not belong to $\mathcal{V}$ because $\exp \left(|x|^{\beta}\right)$ and $\exp \left((|x|+1)^{\beta}\right)$ are not comparable.

The main result of this paper is the following theorem.

Theorem 1.6. Assume that the potential $V$ belongs to class $\mathcal{V}$. Then the FeynmanKac semigroup $T_{t}$ with such potential (defined by (1.1)) is intrinsically ultracontractive if and only if

$$
\lim _{|x| \rightarrow \infty} \frac{V(x)}{|x|}=\infty .
$$

Moreover, if (1.4) holds, then there exist $c_{1}=c_{1}(d, \alpha, m, V)>0$ and $c_{2}=$ $c_{2}(d, \alpha, m, V)>0$ such that for any $x \in \mathbf{R}^{d}$ we have

$$
\frac{c_{1} \exp \left(-m^{1 / \alpha}|x|\right)}{(|x|+1)^{(d+\alpha+1) / 2}(V(x)+1)} \leq \phi_{1}(x) \leq \frac{c_{2} \exp \left(-m^{1 / \alpha}|x|\right)}{(|x|+1)^{(d+\alpha+1) / 2}(V(x)+1)} .
$$

In particular for potentials $V(x)=|x|^{\beta}$, the semigroup $T_{t}$ is IU if and only if $\beta>1$. When $\beta>1$ there exists $c_{1}=c_{1}(\alpha, d, m, \beta)>0$ and $c_{2}=c_{2}(\alpha, d, m, \beta)>0$ such that

$$
\frac{c_{1} \exp \left(-m^{1 / \alpha}|x|\right)}{(|x|+1)^{(d+\alpha+2 \beta+1) / 2}} \leq \phi_{1}(x) \leq \frac{c_{2} \exp \left(-m^{1 / \alpha}|x|\right)}{(|x|+1)^{(d+\alpha+2 \beta+1) / 2}} .
$$

This gives control on the growth of $u(t, x, y)$ (cf. (1.3)) .

The rest of the paper is organized as follows. In Section 2, Preliminaries, we set notation and present various facts which are needed in the sequel. In Section 3 we prove Theorem 1.1 which gives criterion for compactness of $T_{t}$. In Section 4 we prove estimates of transition density for the killed process. These estimates are needed to prove the main result. Nevertheless it seems that these estimates were not known before and are interesting in themselves. In Section 5 we prove the main result of the paper, Theorem[1.6. Section 5 is the most important and difficult part of this paper. We use probabilistic methods to prove intrinsic ultracontractivity. 
One of the key steps in the proof of the main theorem is Lemma 5.9. The main idea of the proof of this lemma is taken from [BK], Lemma 4.5.

\section{Preliminaries}

Let $\mathbb{N}=\{1,2, \ldots\}$ denote the set of natural numbers. Let $d \geq 1$. By $|x|$ we will denote the Euclidean norm of in $\mathbf{R}^{d}$, and by $|A|$ the $d$-dimensional Lebesgue measure of set $A$. For any subset $U \in \mathbf{R}^{d}$ we will denote its complement by $U^{c}$. Furthermore for $x \in \mathbf{R}^{d}, r>0$, we put $B(x, r)=\left\{y \in \mathbf{R}^{d}:|x-y|<r\right\}$. For any $A, B \subset \mathbf{R}^{d}, t>0$, we denote $\operatorname{dist}(A, B)=\inf \{x \in A, y \in B:|x-y|\}$, $t A=\{t x: x \in A\}, \delta_{A}(x)=\operatorname{dist}(x, \partial A)$.

We will write $c=c(\alpha, \beta, \ldots, \gamma)$ to indicate the dependence of a constant $c$ on parameters, functions, etc. All constants in this paper depend on the process, and (if applicable) on the potential, thus we will omit dependence on $\alpha, d, m$, and $V$. The constants may change their value from one use to the next, even on the same line in the same formula. However, the set of parameters on which a constant may depend will not change from one use to another. The constants will always be assumed finite and strictly positive.

From now on let $\alpha \in(0,2)$. We will follow terminology and notation from $\underline{\mathbb{R}}$ most of the time.

The density of the transition probability for $X_{t}$ is given by the formula

$$
\mathbf{P}^{x}\left(X_{t} \in A\right)=\int_{A} p(t, x, y) d y .
$$

It is well known (Lemma 3 from $[\underline{\mathrm{R}}$ ) that for all $t>0$ the density $p(t, x, y)$ is bounded. The density of the Levy measure for the relativistic $\alpha$-stable process, called $\nu(x)$, is equal to (Lemma 2 from $[\underline{\mathrm{R}}$ )

$$
\nu(x)=\frac{c}{|x|^{d+\alpha}} e^{-m^{1 / \alpha}|x|} \varphi\left(m^{1 / \alpha}|x|\right),
$$

for $x \in \mathbf{R}^{d},|x|>0$, where

$$
\varphi(\xi)=\int_{0}^{\infty} e^{-v} v^{p}(\xi+v / 2)^{p} d v, \quad \xi \geq 0, p=\frac{d+\alpha-1}{2},
$$

and $c=\Gamma((d+\alpha) / 2) /\left(\pi^{d / 2} 2^{-\alpha / 2}|\Gamma(-\alpha / 2)| \varphi(0)\right)$.

By $\tau_{D}$ we will denote the first exit time from the open set $D$, i.e., $\tau_{D}=$ $\inf \left\{t>0: X_{t} \notin D\right\}$. The exit time has the following property: $\mathbf{P}^{x}\left(\tau_{D}=t\right)=0$ for all $t>0$.

By $p_{D}(t, x, y)$ we denote the density of the process killed on exiting the set $D$ :

$$
p_{D}(t, x, y)=p(t, x, y)-\mathbf{E}^{x}\left(\tau_{D} \leq t ; p\left(t-\tau_{D}, X\left(\tau_{D}\right), y\right)\right),
$$

for $x, y \in D$, and $p_{D}(t, x, y)=0$ everywhere else. For the open bounded set $D$ we will denote by $G_{D}(x, y)$ the Green function for the set $D$ equal to $G_{D}(x, y)=$ $\int_{0}^{\infty} p_{D}(t, x, y) d t$.

For an open set $D \subset \mathbf{R}^{d}$ and $x \in \mathbf{R}^{d}$, the distribution $\mathbf{P}^{x}\left(\tau_{D}<\infty, X\left(\tau_{D}\right) \in \cdot\right)$ will be called the relativistic $\alpha$-harmonic measure for $D$. The following IkedaWatanabe formula recovers the relativistic $\alpha$-harmonic measure for the set $D$ from the Green function. 
Proposition 2.1 ([W]). Assume that $D$ is an open, nonempty, bounded subset of $\mathbf{R}^{d}$, and $A$ is a Borel set such that $\operatorname{dist}(D, A)>0$. Then

$$
\mathbf{P}^{x}\left(X\left(\tau_{D}\right) \in A, \tau_{D}<\infty\right)=\int_{D} G_{D}(x, y) \int_{A} \nu(y-z) d z d y, x \in D .
$$

Now we prove some estimates for $p(t, x, y)$ and $\nu(x)$, which are crucial in further considerations.

Lemma 2.2. For any $x, y \in \mathbf{R}^{d}$ and $t>0$ there exist constants $c_{1}>0$ and $c_{2}>0$, such that

$$
p(t, x, y) \leq c_{1} e^{m t} \min \left\{\frac{t}{|x-y|^{d+\alpha}} e^{-c_{2}|x-y|}, t^{-d / \alpha}\right\} .
$$

Proof. Let us recall our convention that all constants (in particular $c_{1}, c_{2}$ ) may depend on $\alpha, d, m, V$, and we omit this dependence in notation. Moreover, all constants are strictly positive. The following inequality is the consequence of formula (8) in the proof of Lemma 2 from $[\mathrm{R}]$

$$
p(t, x, y) \leq c t e^{m t} \int_{0}^{\infty} \frac{1}{u^{\frac{d+\alpha+2}{2}}} e^{-m^{2 / \alpha} u} e^{\frac{-|x-y|^{2}}{4 u}} d u .
$$

We have

$$
\begin{aligned}
& \int_{0}^{|x-y|} \frac{1}{u^{\frac{d+\alpha+2}{2}}} e^{-m^{2 / \alpha} u} e^{\frac{-|x-y|^{2}}{4 u}} d u \leq e^{-|x-y| / 8} \int_{0}^{|x-y|} \frac{1}{u^{\frac{d+\alpha+2}{2}}} e^{\frac{-|x-y|^{2}}{8 u}} d u, \\
& \int_{|x-y|}^{\infty} \frac{1}{u^{\frac{d+\alpha+2}{2}}} e^{-m^{2 / \alpha} u} e^{\frac{-|x-y|^{2}}{4 u}} d u \leq e^{-m^{2 / \alpha}|x-y|} \int_{|x-y|}^{\infty} \frac{1}{u^{\frac{d+\alpha+2}{2}}} e^{\frac{-|x-y|^{2}}{8 u}} d u .
\end{aligned}
$$

Thus

$$
p(t, x, y) \leq c t e^{m t} e^{-c_{2}|x-y|} \int_{0}^{\infty} \frac{1}{u^{\frac{d+\alpha+2}{2}}} e^{\frac{-|x-y|^{2}}{8 u}} d u .
$$

Substituting $v=|x-y|^{2} /(8 u)$, the last expression equals

$$
c t e^{m t} e^{-c_{2}|x-y|} \int_{0}^{\infty} \frac{v^{\frac{d+\alpha-2}{2}}}{|x-y|^{d+\alpha}} e^{-v} d v=\frac{c_{1} t e^{m t}}{|x-y|^{d+\alpha}} e^{-c_{2}|x-y|} .
$$

The second estimate (by $t^{-d / \alpha}$ ) is a consequence of Lemma 5 from $[\mathbb{R}$ and wellknown estimates for transition density for classical $\alpha$-stable processes.

We obtained the following explicit formula for the asymptotic behavior of $\nu(x)$.

Lemma 2.3. Let $\varepsilon>0$. There exist constants $c_{\varepsilon}$ and $C_{\varepsilon}$, such that for $|x|>\varepsilon$ we have

$$
\frac{c_{\varepsilon}}{|x|^{\frac{d+\alpha+1}{2}}} e^{-m^{1 / \alpha}|x|} \leq \nu(x) \leq \frac{C_{\varepsilon}}{|x|^{\frac{d+\alpha+1}{2}}} e^{-m^{1 / \alpha}|x|} .
$$

Proof. To get the asymptotic behavior of $\nu(x)$ we need to estimate $\varphi$ from (2.2). Assume $\xi \geq \varepsilon m^{1 / \alpha}$. We divide $\varphi$ into two parts,

$$
\begin{gathered}
I_{1}=\int_{0}^{\xi} e^{-v} v^{p}(\xi+v / 2)^{p} d v, \\
I_{2}=\int_{\xi}^{\infty} e^{-v} v^{p}(\xi+v / 2)^{p} d v .
\end{gathered}
$$


We have

$$
I_{2} \leq \int_{\xi}^{\infty} e^{-v} v^{p}(2 v)^{p} d v \leq 2^{p} \int_{0}^{\infty} e^{-v} v^{2 p} d v,
$$

so $I_{2}$ is bounded. On the other hand,

$$
\begin{aligned}
& I_{1} \leq(2 \xi)^{p} \int_{0}^{\xi} e^{-v} v^{p} d v \leq(2 \xi)^{p} \int_{0}^{\infty} e^{-v} v^{p} d v=c|\xi|^{p}, \\
& I_{1} \geq|\xi|^{p} \int_{0}^{\xi} e^{-v} v^{p} d v \geq|\xi|^{p} \int_{0}^{\varepsilon m^{1 / \alpha}} e^{-v} v^{p} d v=c|\xi|^{p} .
\end{aligned}
$$

Lemma 2.4. For any $x \in \mathbf{R}^{d}$ we have

$$
\nu(x) \leq \frac{c}{|x|^{d+\alpha}} .
$$

Proof. The lemma is a consequence of formulas (2.1) and (2.2).

Now we prove generalizations of the Ikeda-Watanabe formula (Proposition 2.1).

Proposition 2.5. Assume that $D$ is an open, nonempty, bounded subset of $\mathbf{R}^{d}$, and $A$ is a Borel set such that $A \subset D^{c} \backslash \partial D$. Then

$$
\begin{aligned}
\mathbf{P}^{x}\left(X\left(\tau_{D}\right) \in A,\right. & \left.t_{1}<\tau_{D}<t_{2}\right) \\
= & \int_{D} \int_{t_{1}}^{t_{2}} p_{D}(s, x, y) d s \int_{A} \nu(y-z) d z d y,
\end{aligned}
$$

where $x \in D, 0 \leq t_{1}<t_{2} \leq \infty$.

Proof. It is sufficient to consider only the case when $t_{2}=\infty$. At first assume that $\operatorname{dist}(A, D)>0$. Using strong Markov property we have, for any $t>0$ and $x \in D$,

$$
\begin{aligned}
\mathbf{P}^{x}\left(X\left(\tau_{D}\right)\right. & \left.\in A, t<\tau_{D}<\infty\right) \\
& =\mathbf{E}^{x}\left(t<\tau_{D} ; \mathbf{P}^{X_{t}}\left(X\left(\tau_{D}\right) \in A, \tau_{D}<\infty\right)\right) \\
& =\mathbf{E}^{x}\left(t<\tau_{D} ; \int_{D} G_{D}\left(X_{t}, y\right) \int_{A} \nu(y-z) d z d y\right) \\
& =\mathbf{E}^{x}\left(t<\tau_{D} ; \int_{D} \int_{0}^{\infty} p_{D}\left(s, X_{t}, y\right) d s \int_{A} \nu(y-z) d z d y\right) \\
& =\int_{D} \int_{0}^{\infty} \mathbf{E}^{x}\left(t<\tau_{D} ; p_{D}\left(s, X_{t}, y\right)\right) d s \int_{A} \nu(y-z) d z d y \\
& =\int_{D} \int_{0}^{\infty} \int_{\mathbf{R}^{d}} p_{D}(t, x, z) p_{D}(s, z, y) d z d s \int_{A} \nu(y-z) d z d y .
\end{aligned}
$$

Now using the semigroup property for $p_{D}$ (Theorem 1 from $[\mathrm{R}]$ ) and changing the limits in the second integral, this is equal to

$$
\int_{D} \int_{t}^{\infty} p_{D}(s, x, y) d s \int_{A} \nu(y-z) d z d y .
$$

If $\operatorname{dist}(A, D)=0$ let us put $A_{\epsilon}=\{z \in A: \operatorname{dist}(z, D)>\epsilon\}, \epsilon>0$. Then (2.7) holds for $A_{\epsilon}$. Letting $\epsilon \rightarrow 0$ we get (2.7) for $A$.

To prove the next generalization we need the following fact. 
Lemma 2.6. For any open nonempty set $D$, and $D_{R}=D \cap B(0, R)$,

$$
p_{D_{R}}(t, x, y) \nearrow p_{D}(t, x, y)
$$

as $R \longrightarrow \infty$, for any $x, y \in D, t>0$.

Proof. We choose $R$ large enough so that $x, y \in D_{R}$. By the definition of $p_{D}$ (2.3), $p_{D}(t, x, y)-p_{D_{R}}(t, x, y)$ is equal to

$$
\mathbf{E}^{x}\left(\tau_{D_{R}}<t ; p\left(t-\tau_{D_{R}}, X\left(\tau_{D_{R}}\right), y\right)\right)-\mathbf{E}^{x}\left(\tau_{D}<t ; p\left(t-\tau_{D}, X\left(\tau_{D}\right), y\right)\right) .
$$

Note that $\tau_{D_{R}} \leq \tau_{D}$ and

$$
\begin{aligned}
& \mathbf{E}^{x}\left(\tau_{D}<t, \tau_{D_{R}}=\tau_{D} ; p\left(t-\tau_{D}, X\left(\tau_{D}\right), y\right)\right) \\
& \quad=\mathbf{E}^{x}\left(\tau_{D_{R}}<t, \tau_{D_{R}}=\tau_{D} ; p\left(t-\tau_{D_{R}}, X\left(\tau_{D_{R}}\right), y\right)\right) .
\end{aligned}
$$

Therefore (2.8) is equal to

$$
\begin{aligned}
& \mathbf{E}^{x}\left(\tau_{D_{R}}<t,\right.\left.\tau_{D_{R}}<\tau_{D} ; p\left(t-\tau_{D_{R}}, X\left(\tau_{D_{R}}\right), y\right)\right) \\
& \quad-\mathbf{E}^{x}\left(\tau_{D_{R}}<\tau_{D}<t ; p\left(t-\tau_{D}, X\left(\tau_{D}\right), y\right)\right) \\
& \leq \mathbf{E}^{x}\left(\tau_{D_{R}}<t, \tau_{D_{R}}<\tau_{D} ; p\left(t-\tau_{D_{R}}, X\left(\tau_{D_{R}}\right), y\right)\right) \\
&=\mathbf{E}^{x}\left(\tau_{D_{R}}<t, X\left(\tau_{D_{R}}\right) \in D ; p\left(t-\tau_{D_{R}}, X\left(\tau_{D_{R}}\right), y\right)\right) .
\end{aligned}
$$

Note also that if $X\left(\tau_{D_{R}}\right) \in D$, then $X\left(\tau_{D_{R}}\right) \in B^{c}(0, R)$. Thus using Lemma 2.2 the last expression is bounded from the above by

$$
\begin{aligned}
& \mathbf{E}^{x}\left(\tau_{D_{R}}<t, X\left(\tau_{D_{R}}\right) \in D ; \frac{c_{1}\left(t-\tau_{D_{R}}\right) e^{m\left(t-\tau_{D_{R}}\right)}}{\left|X\left(\tau_{D_{R}}\right)-y\right|^{d+\alpha}} e^{-c_{2}\left|X\left(\tau_{D_{R}}\right)-y\right|}\right) \\
& \quad \leq \frac{c_{1} t e^{m t}}{(R-|y|)^{d+\alpha}} e^{-c_{2}(R-|y|)} .
\end{aligned}
$$

The last expression tends to 0 as $R \longrightarrow \infty$, thus the lemma is proved.

Proposition 2.7. Assume that $D$ is open and nonempty (it may be unbounded) and $A$ is a Borel set such that $A \subset D^{c} \backslash \partial D$. Also assume that $0 \leq t_{1}<t_{2}<\infty$. Then (2.7) holds.

Proof. Without loss of generality we can assume that $t_{1}=0$. Consider the family of sets $D_{R}=D \cap B(0, R), R>0$. These sets are open, bounded and nonempty for large enough $R$, so we may apply Proposition 2.5 to those sets and $t_{2}=t \geq 0$ :

$$
\mathbf{P}^{x}\left(X\left(\tau_{D_{R}}\right) \in A, \tau_{D_{R}}<t\right)=\int_{D_{R}} \int_{0}^{t} p_{D_{R}}(s, x, y) d s \int_{A} \nu(y-z) d z d y .
$$

The proof will be completed if we show that

$$
\begin{gathered}
\mathbf{P}^{x}\left(X\left(\tau_{D_{R}}\right) \in A, \tau_{D_{R}}<t\right) \longrightarrow \mathbf{P}^{x}\left(X\left(\tau_{D}\right) \in A, \tau_{D}<t\right), \\
p_{D_{R}}(s, x, y) \nearrow p_{D}(s, x, y),
\end{gathered}
$$

as $R \longrightarrow \infty$. 
Lemma 2.6 gives (2.11). We need to show (2.10). We may and do assume that $x \in D_{R}$. Note that if $X\left(\tau_{D_{R}}\right) \in A$, then $\tau_{D_{R}}=\tau_{D}$. Thus

$$
\begin{aligned}
\mathbf{P}^{x}\left(X\left(\tau_{D}\right)\right. & \left.\in A, \tau_{D}<t\right)-\mathbf{P}^{x}\left(X\left(\tau_{D_{R}}\right) \in A, \tau_{D_{R}}<t\right) \\
& =\mathbf{P}^{x}\left(X\left(\tau_{D}\right) \in A, X\left(\tau_{D_{R}}\right) \in D, \tau_{D_{R}}<\tau_{D}<t\right) \\
& \leq \mathbf{P}^{x}\left(X\left(\tau_{D_{R}}\right) \in D \backslash B(0, R), \tau_{D_{R}}<t\right) \\
& =\mathbf{P}^{x}\left(X\left(\tau_{D_{R}}\right) \in D \backslash B(0, R), \tau_{D_{R}}<t, \tau_{B(0, R)}<t\right) \\
& \leq \mathbf{P}^{x}\left(\tau_{B(0, R)}<t\right) .
\end{aligned}
$$

For $R>2|x|$ this is bounded from above by

$$
\begin{aligned}
& \mathbf{P}^{x}\left(\tau_{B(x, R / 2)}<t\right)=\mathbf{P}^{0}\left(\tau_{B(0, R / 2)}<t\right) \\
& \quad=1-P^{0}\left(\tau_{B(0, R / 2)}>t\right)=1-\int_{\mathbf{R}^{d}} p_{B(0, R / 2)}(t, 0, y) d y .
\end{aligned}
$$

By Lemma 2.6 this decreases to $1-\int_{\mathbf{R}^{d}} p(t, 0, y) d y=0$.

The above proposition gives an explicit formula for the joint distribution of $X\left(\tau_{D}\right)$ and $\tau_{D}$, thus as an easy consequence we have

Corollary 2.8. Assume that $D$ is an open and nonempty set (it may be unbounded) and $A$ is a Borel set such that $A \subset D^{c} \backslash \partial D$. Also assume that $0 \leq t<\infty$ and $B$ is any Borel set. Then

$$
\begin{aligned}
\mathbf{P}^{x}\left(X\left(\tau_{D}\right)\right. & \left.\in A, \tau_{D}<t, X(t) \in B\right) \\
& =\int_{D} \int_{0}^{t} p_{D}(s, x, y) \int_{A} \nu(y-z) \mathbf{P}^{z}(X(t-s) \in B) d z d s d y .
\end{aligned}
$$

In the sequel we will need another generalization of the Ikeda-Watanabe formula. Namely we need to change the assumption $A \subset D^{c} \backslash \partial D$ to the assumption $A \subset D^{c}$. It is possible to do so, but we have to make some additional regularity assumptions on $\partial D$.

We say that an open set $D \subset \mathbf{R}^{d}$ satisfies the outer cone property if there exist constants $\eta=\eta(D), R_{0}=R_{0}(D)$ and a cone $C=\left\{x=\left(x_{1}, \ldots, x_{d}\right) \in \mathbf{R}^{d}: 0<\right.$ $\left.x_{d},\left\|\left(x_{1}, \ldots, x_{d-1}\right)\right\|<\eta x_{d}\right\}$ such that for every $Q \in \partial D$, there is a cone $C_{Q}$ with vertex $Q$, isometric with $C$ and satisfying $C_{Q} \cap B\left(Q, R_{0}\right) \subset D^{c}$.

For such sets we will be able to prove that $P^{x}\left(X\left(\tau_{D}\right) \in \partial D ; \tau_{D}<\infty\right)=0$, $x \in D$.

At first we need the following auxiliary lemma. We point out that this lemma would be trivial for the symmetric stable process because of the scaling properties of the process. For the relativistic process the proof requires more technical details.

Lemma 2.9. Let $D$ be an open, nonempty, bounded set satisfying the outer cone property. For $x \in D$ let $r_{x}=\frac{1}{3} \operatorname{dist}\left(x, D^{c}\right)$ and $B_{x}=B\left(x, r_{x}\right)$. There exists a constant $p=p(D)>0$ (not depending on $x$ ) such that for any $x \in D$ we have

$$
P^{x}\left(X\left(\tau_{B_{x}}\right) \in D^{c}\right)>p .
$$

Proof. Let $x \in D$. By Lemma 7 from $[\underline{\mathrm{R}}$ we have

$$
E^{x}\left(\tau_{B_{x}}\right) \geq\left(1+m c|D|^{\alpha / d}\right)^{-1} E^{x}\left(\tilde{\tau}_{B_{x}}\right),
$$

where $\tilde{\tau}_{B_{x}}$ is the exit time for the symmetric $\alpha$-stable process and $c$ a constant depending only on $d$ and $\alpha$. It is well known (see e.g. [BK] (2.10)) that $E^{x}\left(\tilde{\tau}_{B_{x}}\right)=$ 
$c^{\prime} r_{x}^{\alpha}$, where $c^{\prime}$ depends only on $d$ and $\alpha$. Therefore $E^{x}\left(\tau_{B_{x}}\right) \geq c r_{x}^{\alpha}$, where $c=c(D)$ (recall that we omit the dependence on $\alpha, d, m$ in the notation). Let $Q \in \partial D$ be such that $\operatorname{dist}(x, \partial D)=|Q-x|$. We have $C_{Q} \cap B\left(Q, R_{0}\right) \subset D^{c}$. By Proposition 2.1 and formula (2.1) we get

$$
\begin{aligned}
& P^{x}\left(X\left(\tau_{B_{x}}\right) \in D^{c}\right)=\int_{B_{x}} G_{B_{x}}(x, y) \int_{D^{c}} \nu(y-z) d z d y \\
& \geq \int_{B_{x}} G_{B_{x}}(x, y) \int_{C_{Q} \cap B\left(Q, R_{0}\right)} c|y-z|^{-d-\alpha} e^{-m^{1 / \alpha}|y-z|} \varphi\left(m^{1 / \alpha}|y-z|\right) d z d y .
\end{aligned}
$$

Note that for $y \in B_{x}$ and $z \in C_{Q} \cap B\left(Q, R_{0}\right)$ we have $|y-z| \leq \operatorname{diam}(D)+R_{0}$ and $|y-z| \leq|y-x|+|x-z| \leq 2|x-z|$. We estimate the terms in the integral over $C_{Q} \cap B\left(Q, R_{0}\right)$ as $|y-z|^{-d-\alpha} \geq c|x-z|^{-d-\alpha}, e^{-m^{1 / \alpha}|y-z|} \geq c, c=c(D)$. By (2.2) $\varphi(\xi) \geq c$ for any $\xi \geq 0 . \int_{B_{x}} G_{B_{x}}(x, y) d y=E^{x}\left(\tau_{B_{x}}\right) \geq c r_{x}^{\alpha}, c=c(D)$. Therefore using (2.13) we obtain

$$
P^{x}\left(X\left(\tau_{B_{x}}\right) \in D^{c}\right) \geq c r_{x}^{\alpha} \int_{C_{Q} \cap B\left(Q, R_{0}\right)}|x-z|^{-d-\alpha} d z, \quad c=c(D) .
$$

We will consider 2 cases, $r_{x} \geq R_{0} / 2$ and $r_{x}<R_{0} / 2$.

If $r_{x} \geq R_{0} / 2$, then the estimate is easy. Note that $r_{x}^{\alpha} \geq\left(R_{0} / 2\right)^{\alpha}$ and $|x-z| \leq$ $\operatorname{diam}(D)+R_{0}$, so the right-hand side of (2.14) is bounded from below by $c(D)$.

If $r_{x}<R_{0} / 2$ put $C_{Q}^{\prime}=C_{Q} \cap B\left(Q, R_{0}\right) \cap B^{c}\left(Q, r_{x}\right)\left(z \in C_{Q}^{\prime}\right.$ if $z \in C_{Q}$ and $r_{x} \leq$ $\left.|z-Q| \leq R_{0}\right)$. For $z \in C_{Q}^{\prime}$ we have $|x-z| \leq|x-Q|+|Q-z|=3 r_{x}+|Q-z| \leq 4|Q-z|$. Therefore

$$
\int_{C_{Q}^{\prime}}|x-z|^{-d-\alpha} d z \geq c \int_{C_{Q}^{\prime}}|Q-z|^{-d-\alpha} d z=c(D) r_{x}^{-\alpha}
$$

Lemma 2.10. Let $D$ be an open, nonempty set (it may be unbounded) satisfying the outer cone property. Then for any $x \in D$ we have

$$
P^{x}\left(X\left(\tau_{D}\right) \in \partial D ; \tau_{D}<\infty\right)=0 .
$$

Proof. At first we note that $|\partial D|=0$ (the Lebesgue measure). This follows from the fact that almost every point of a measurable set is a density point. Since for $x \in \partial D$ there exists a cone belonging to $D^{c}$, the point $x$ is not a density point for $\partial D$. Therefore $|\partial D|=0$.

In the next step let us assume that $D$ is bounded. Then we may repeat the proof which is given in $\mathrm{Bo}$, Lemma 6 , for symmetric $\alpha$-stable processes. Some changes in the proof are needed because we have to prove it for relativistic stable processes which do not have nice scaling properties. Therefore we will repeat the main steps from the proof of $[\mathrm{Bo}$, Lemma 6 .

By Theorems 3 and 2 from $\left[\underline{\mathrm{R}}\right.$ we have $E^{x}\left(\tau_{B(0, R)}\right)<\infty$ for any $R>0$ and $x \in$ $B(0, R)$. Since $D$ is bounded it follows that $E^{x}\left(\tau_{D}\right)<\infty$, hence $P^{x}\left(\tau_{D}<\infty\right)=1$, for any $x \in D$. Therefore we will omit $\tau_{D}<\infty$ and write $P^{x}\left(X\left(\tau_{D}\right) \in \partial D\right)$ instead of $P^{x}\left(X\left(\tau_{D}\right) \in \partial D ; \tau_{D}<\infty\right)$ whenever $D$ is bounded. 
For $x \in D$ let $r_{x}=\frac{1}{3} \operatorname{dist}\left(x, D^{c}\right)$ and $B_{x}=B\left(x, r_{x}\right)$. By the strong Markov property we have for $x \in D$

$$
\begin{aligned}
& P^{x}\left(X\left(\tau_{D}\right) \in \partial D\right) \\
& =P^{x}\left(X\left(\tau_{B_{x}}\right) \in \partial D\right)+E^{x}\left(P^{X\left(\tau_{B_{x}}\right)}\left(X\left(\tau_{D}\right) \in \partial D\right) ; X\left(\tau_{B_{x}}\right) \in D\right) .
\end{aligned}
$$

We denote the two terms on the right-hand side of (2.15) by $p_{0}(x)$ and $r_{0}(x)$, respectively. We observe that $p_{0}(x)$ is the probability of the event that the process $X_{t}$ jumps directly to $\partial D$ while leaving $B_{x}$, and $r_{0}(x)$ is the probability of a complimentary event that upon leaving $B_{x}$ it visits $D$ before going to $\partial D$.

Using (2.15) we can prove inductively that for $k=0,1, \ldots$ we have

$$
P^{x}\left(X\left(\tau_{D}\right) \in \partial D\right)=p_{0}(x)+p_{1}(x)+\ldots+p_{k}(x)+r_{k}(x), \quad x \in D,
$$

with

$$
p_{k+1}(x)=E^{x}\left(p_{k}\left(X\left(\tau_{B_{x}}\right)\right) ; X\left(\tau_{B_{x}}\right) \in D\right)
$$

and

$$
r_{k+1}(x)=E^{x}\left(r_{k}\left(X\left(\tau_{B_{x}}\right)\right) ; X\left(\tau_{B_{x}}\right) \in D\right) .
$$

Indeed, it is enough to verify that $r_{k}=p_{k+1}+r_{k+1}$. We may think of $p_{k}$ as the probability of the event that the process $X_{t}$ goes to $\partial D$ after precisely $k$ jumps from one ball $B_{x}$ to another.

Recall that we assume that $D$ is bounded so we can use Lemma 2.9, By this lemma and (2.18) we get

$$
\sup _{x \in D} r_{k+1}(x) \leq(1-p) \sup _{x \in D} r_{k}(x) \leq(1-p)^{k+1} \rightarrow 0 \quad \text { as } \quad k \rightarrow \infty .
$$

By (2.16) we obtain $P^{x}\left(X\left(\tau_{D}\right) \in \partial D\right)=\sum_{k=0}^{\infty} p_{k}(x)$.

Now note that for any $x \in D$, $\operatorname{dist}\left(B_{x}, \partial D\right)>0$, so we may apply Proposition 2.1. But $|\partial D|=0$, so this proposition gives that $p_{0}(x)=0$ for all $x \in D$. By (2.17) we obtain that $p_{k}(x)=0$ for all $x \in D$ and all $k$. Therefore $P^{x}\left(X\left(\tau_{D}\right) \in \partial D\right)=0$.

Next let us assume that $D$ is unbounded. Let $D_{n}=D \cap B(0, n), n=1,2, \ldots$. For $x \in D_{n}$ we have

$$
P^{x}\left(X\left(\tau_{D_{n}}\right) \in \partial D\right)=P^{x}\left(X\left(\tau_{D_{n}}\right) \in \partial D_{n} \cap \partial D\right)+P^{x}\left(X\left(\tau_{D_{n}}\right) \in \partial D \backslash \partial D_{n}\right) .
$$

The first term on the right-hand side is 0 because $D_{n}$ satisfies the outer cone property and is bounded. The second term is 0 by Proposition 2.5 (for $t_{1}=0$, $\left.t_{2}=\infty\right)$ and the fact that $|\partial D|=0$.

Now we will use a very general fact that for each fixed $\omega \in \Omega$ and $t>0$, we have $\sup _{s \in[0, t]}\left|X_{s}(\omega)\right|<\infty$. Therefore for any fixed $\omega \in \Omega$ such that $\tau_{D}(\omega)<\infty$, we have $\sup _{s \in\left[0, \tau_{D}(\omega)\right]} X_{s}(\omega)<\infty$. Hence for any fixed $\omega \in \Omega$ such that $\tau_{D}(\omega)<\infty$, there exists $n=n(\omega)$ such that $\tau_{D}(\omega)=\tau_{D_{n}}(\omega)$. It follows that

$$
P^{x}\left(X\left(\tau_{D}\right) \in \partial D ; \tau_{D}<\infty\right)=\lim _{n \rightarrow \infty} P^{x}\left(X\left(\tau_{D_{n}}\right) \in \partial D\right)=0 .
$$

As an immediate conclusion of Corollary 2.8 and Lemma 2.10 we obtain the following corollary.

Corollary 2.11. Assume that $D$ is an open and nonempty set satisfying the outer cone property (it may be unbounded) and $A$ is a Borel set such that $A \subset D^{c}$. Also assume that $0 \leq t<\infty$ and $B$ is any Borel set. Then (2.12) holds. 


\section{Compactness of the Feynman-KaC semigroup}

At the beginning of this section we prove the existence and basic properties of the kernel $u(t, x, y)$. The proof is standard and is based on CZ (see Section 3.2). Let us denote $P_{t} f(x)=E^{x}\left(f\left(X_{t}\right)\right)$. Using estimates for $p(t, x, y)$ (see Lemma $2.2)$ it is easy to show that $P_{t}: L^{1}\left(\mathbf{R}^{d}\right) \rightarrow L^{\infty}\left(\mathbf{R}^{d}\right), P_{t}: L^{1}\left(\mathbf{R}^{d}\right) \rightarrow L^{1}\left(\mathbf{R}^{d}\right)$ and $P_{t}: L^{\infty}\left(\mathbf{R}^{d}\right) \rightarrow L^{\infty}\left(\mathbf{R}^{d}\right)$ are bounded operators. By $C B\left(\mathbf{R}^{d}\right)$ we denote the set of all continuous and bounded functions on $\mathbf{R}^{d}$.

Lemma 3.1. (i) $T_{t}(|f|)(x) \leq P_{t}(|f|)(x), t>0, x \in \mathbf{R}^{d}, f: \mathbf{R}^{d} \rightarrow \mathbf{R}$.

(ii) For any $t>0, T_{t}: L^{\infty} \rightarrow C B\left(\mathbf{R}^{d}\right)$.

(iii) There exists a density $u(t, x, y)$ for $T_{t}$, i.e. $T_{t} f(x)=\int u(t, x, y) f(y) d y$, $t>0, x \in \mathbf{R}^{d}, f \in L^{p}\left(\mathbf{R}^{d}\right)(1 \leq p \leq \infty)$. For each fixed $t>0, u(t, x, y)$ is continuous and bounded on $\mathbf{R}^{d} \times \mathbf{R}^{d}$.

(iv) $u(t, x, y)=u(t, y, x), t>0, x, y \in \mathbf{R}^{d}$.

(v) $u(t, x, y) \leq p(t, x, y), t>0, x, y \in \mathbf{R}^{d}$.

(vi) $u(t, x, y)>0, t>0, x, y \in \mathbf{R}^{d}$.

Proof. (i) This is clear from (1.1) and our assumption that $V \geq 0$.

(ii) Put $u(x, y)=\int_{0}^{\infty} p(s, x, y) d s, x, y \in \mathbf{R}^{d}$. By $\mathcal{J}$ we denote the Kato class for the relativistic $\alpha$-stable process. We say that $q: \mathbf{R}^{d} \rightarrow \mathbf{R}$ belongs to $\mathcal{J}$ if and only if

$$
\lim _{r \downarrow 0}\left[\sup _{x \in \mathbf{R}^{d}} \int_{|y-x| \leq r} u(x, y)|q(y)| d y\right]=0 .
$$

This definition is motivated by [Z], Theorem 1 (C1). The condition (3.1) implies

$$
\lim _{t \downarrow 0}\left[\sup _{x \in \mathbf{R}^{d}} E^{x}\left(\int_{0}^{t}\left|q\left(X_{s}\right)\right| d s\right)\right]=0 .
$$

This follows from Lemma 2 in [Z] and proof of Theorem 1, steps 4 and 2 in [Z]. Put $e_{q}(t)=\exp \left(\int_{0}^{t} q\left(X_{s}\right) d s\right)$. By a standard argument based on the Khasminskii lemma (see Lemma 3.7 and Proposition 3.8 in [CZ] (3.2) implies

$$
\lim _{t \downarrow 0}\left[\sup _{x \in \mathbf{R}^{d}} E^{x}\left(e_{|q|}(t)\right)\right]=1 .
$$

Now for any $R>0, x \in \mathbf{R}^{d}$ put $V_{R}(x)=1_{B(0, R)}(x) V(x)$. Recall our assumption that $d>\alpha$ and $V$ is locally bounded. Estimates of $u(x, y)$ from Lemma 4 in $[\mathrm{R}$ ] give that $V_{R} \in \mathcal{J}$ for any $R>0$. Set $T_{t, R} f(x)=E^{x}\left(e_{-V_{R}}(t) f\left(X_{t}\right)\right), t>0, R>0$, $x \in \mathbf{R}^{d}$. Formula (7) from $[\mathrm{R}]$ implies that for each fixed $t>0$ the kernel $p(t, x, y)$ is bounded and continuous on $\mathbf{R}^{d} \times \mathbf{R}^{d}$. It follows that $P_{t}: L^{\infty}\left(\mathbf{R}^{d}\right) \rightarrow C B\left(\mathbf{R}^{d}\right)$. Using this, formula (3.3) and the same arguments as in the proofs of Propositions 3.11 and 3.12 from $\mathrm{CZ}$, we obtain that $T_{t, R}: L^{\infty}\left(\mathbf{R}^{d}\right) \rightarrow C B\left(\mathbf{R}^{d}\right)$. We also have

$$
\left|T_{t} f(x)-T_{t, R} f(x)\right|=\left|E^{x}\left(\left(e_{-V}(t)-e_{-V_{R}}(t)\right) f\left(X_{t}\right)\right)\right| \leq\|f\|_{\infty} P^{x}\left(\tau_{B(0, R)}<t\right) .
$$

For each fixed $t>0, P^{x}\left(\tau_{B(0, R)}<t\right)$ tends to 0 as $R$ tends to $\infty$. This implies (ii).

(iii)-(v) By (i) and properties of $P_{t}$ we obtain $T_{t}: L^{1}\left(\mathbf{R}^{d}\right) \rightarrow L^{\infty}\left(\mathbf{R}^{d}\right)$ and $T_{t}: L^{1}\left(\mathbf{R}^{d}\right) \rightarrow L^{1}\left(\mathbf{R}^{d}\right)$ are bounded operators. It follows from this and a theorem due to Dunford and Pettis (see [S], Theorem A.1.1, Corollary A.1.2) (cf. also [CZ], page 77 ) that for each $t>0$ there exists a measurable (on $\mathbf{R}^{d} \times \mathbf{R}^{d}$ ) density $u(t, x, y)$, 
$x, y \in \mathbf{R}^{d}$ for $T_{t}$, i.e.

$$
T_{t} f(x)=\int u(t, x, y) f(y) d y, \quad f \in L^{1}\left(\mathbf{R}^{d}\right), t>0, x \in \mathbf{R}^{d} .
$$

In fact, by (i) and properties of $P_{t}$ it is not difficult to show that this formula holds for all $f \in L^{p}(1 \leq p \leq \infty)$.

(i) and (1.1) imply that for each fixed $t>0$ and $x \in \mathbf{R}^{d}$ we have $0 \leq u(t, x, y) \leq$ $p(t, x, y)$ for almost all $y \in \mathbf{R}^{d}$. We may and do assume that these two inequalities hold for all $y \in \mathbf{R}^{d}$. In particular this gives (v).

By standard arguments (see [CZ, pages 75-76) $T_{t}$ is symmetric, so for each fixed $t>0$ (iv) holds for almost all (x,y) according to the Lebesgue measure on $\mathbf{R}^{d} \times \mathbf{R}^{d}$.

Put $f_{t, x}(y)=u(t, x, y)$. Fix $t>0, x_{0}, y_{0} \in \mathbf{R}^{d}, r>0$. Using (iv) (for almost all $\left.(x, y) \in \mathbf{R}^{d} \times \mathbf{R}^{d}\right)$ and the semigroup property we get

$$
\int_{B\left(y_{0}, r\right)} u\left(t, x_{0}, y\right) d y=\int_{B\left(y_{0}, r\right)} T_{t / 2} f_{t / 2, x_{0}}(y) d y .
$$

$f_{t / 2, x_{0}} \in L^{\infty}\left(\mathbf{R}^{d}\right)$, so (ii) implies that $T_{t / 2} f_{t / 2, x_{0}} \in C B\left(\mathbf{R}^{d}\right)$. Therefore we may and do assume that for each fixed $t>0$ and $x \in \mathbf{R}^{d}, u(t, x, y)$ is continuous as a function of $y$.

Fixed $t>0$. For any $x, y \in \mathbf{R}^{d}$ we have

$$
u(t, x, y)=\iint u(t / 3, x, z) u(t / 3, z, w) u(t / 3, w, y) d w d z .
$$

For any $z, w \in \mathbf{R}^{d}, u(t / 3, z, x) \rightarrow u\left(t / 3, z, x_{0}\right)$ and $u(t / 3, w, y) \rightarrow u\left(t / 3, w, y_{0}\right)$ when $x \rightarrow x_{0}$ and $y \rightarrow y_{0}$. Using the bounded convergence theorem we get (iii). It follows that (iv) holds for all $t>0, x, y \in \mathbf{R}^{d}$.

(vi) By (1.1) and the fact that $V$ is locally bounded, we obtain that for each fixed $t>0, x \in \mathbf{R}^{d}, u(t, x, y)>0$ for almost all $y \in \mathbf{R}^{d}$ according to the Lebesgue measure. We also have $u(t, x, y)=\int u(t / 2, x, z) u(t / 2, z, y) d z$, so (vi) holds for all $t>0, x, y \in \mathbf{R}^{d}$.

Now we will prove Theorem 1.1. We define an auxiliary operator.

Definition 3.2. Fix $t>0$. For any bounded Borel $A \subset \mathbf{R}^{d}$ let

$$
S_{A}(f)(x)=\int_{A} u(t, x, y) f(y) d y, \quad f \in L^{2}\left(\mathbf{R}^{d}\right) .
$$

Lemma 3.3. For any fixed $t>0$ and any bounded Borel $A \subset \mathbf{R}^{d}$, the operator $S_{A}$ is compact.

Proof. It is sufficient to show that this operator is the Hilbert-Schmidt operator. $K(x, y)=1_{A}(y) u(t, x, y)$ is the integral kernel of $S_{A}$. Using the previous lemma and boundedness of $p(t, x, y)$ (Lemma 2.2) we get

$$
\begin{aligned}
\int_{\mathbf{R}^{d}} \int_{\mathbf{R}^{d}} K^{2}(x, y) d x d y & =\int_{\mathbf{R}^{d}} \int_{A} u^{2}(t, x, y) d y d x \leq \int_{\mathbf{R}^{d}} \int_{A} p^{2}(t, x, y) d y d x \\
& \leq c_{t} \int_{A} \int_{\mathbf{R}^{d}} p(t, x, y) d y d x=c_{t}|A|<\infty .
\end{aligned}
$$

Thus $A$ is the Hilbert-Schmidt operator, so it is compact. 
Lemma 3.4. Assume that $\lim _{|x| \rightarrow \infty} V(x)=\infty$. Fix $t>0$. Then we have

$$
\lim _{R \longrightarrow \infty} \sup _{y \in B^{c}(0, R)} T_{t}\left(1_{\mathbf{R}^{d}}\right)(y)=0 .
$$

Proof. Let $M=\inf _{x \in B^{c}(0, R-1)} V(x)$. Obviously $M \longrightarrow \infty$ as $R \longrightarrow \infty$. Let $\tau=\tau_{B(y, 1)}$.

$$
\begin{aligned}
\sup _{y \in B^{c}(0, R)} T_{t}\left(1_{\mathbf{R}^{d}}\right)(y) & \leq \sup _{y \in B^{c}(0, R)} \mathbf{E}^{y}\left(\exp \left(-\int_{0}^{\min \{t, \tau\}} V\left(X_{s}\right) d s\right)\right) \\
& =\sup _{y \in B^{c}(0, R)}\left\{\mathbf{E}^{y}\left(\tau>t ; \exp \left(-\int_{0}^{t} V\left(X_{s}\right) d s\right)\right)\right. \\
& +\mathbf{E}^{y}\left(\frac{t}{\sqrt{M}}<\tau<t ; \exp \left(-\int_{0}^{\tau} V\left(X_{s}\right) d s\right)\right) \\
& \left.+\mathbf{E}^{y}\left(\tau<\frac{t}{\sqrt{M}} ; \exp \left(-\int_{0}^{\tau} V\left(X_{s}\right) d s\right)\right)\right\} \\
& \leq e^{-M t}+e^{-\sqrt{M} t}+\mathbf{P}^{0}\left(\tau_{B(0,1)}<\frac{t}{\sqrt{M}}\right) .
\end{aligned}
$$

The lemma follows because the last expression tends to 0 as $R \longrightarrow \infty$.

Definition 3.5. The $\varepsilon$-net, $\varepsilon>0$, for the operator $T: L^{2}\left(\mathbf{R}^{d}\right) \longrightarrow L^{2}\left(\mathbf{R}^{d}\right)$ is a finite set $N_{\varepsilon}$ of a function from $L^{2}\left(\mathbf{R}^{d}\right)$, such that for any $f \in L^{2}\left(\mathbf{R}^{d}\right)$ with $\|f\|_{2} \leq 1$ there exists $g \in N_{\varepsilon}$ such that

$$
\|T(f)-g\|_{2}<\varepsilon .
$$

It is easy to see that operator is compact iff for every $\varepsilon>0$, there exists the $\varepsilon$-net for this operator.

Proof of Theorem 1.1. Recall that $V \geq 0$. Assume that $V(x) \rightarrow \infty$ as $|x| \rightarrow \infty$, and fix $t>0$ and $\varepsilon>0$. We will show that there exists the $\varepsilon$-net for $T_{t}$. Choose $R>0$ such that $\sup _{y \in B^{c}(0, R)} T_{t}\left(1_{\mathbf{R}^{d}}\right)(y)<(\varepsilon / 2)^{2}$. It is possible due to Lemma 3.4. Put $A=B^{c}(0, R)$. The operator $S_{A^{c}}$ is compact, so there exists the $(\varepsilon / 2)$-net for this operator. Let us denote this net by $N_{\varepsilon / 2}$. We will show that $N_{\varepsilon / 2}$ is the $\varepsilon$-net for $T_{t}$. Fix arbitrary $f \in L^{2}\left(\mathbf{R}^{d}\right),\|f\|_{2} \leq 1$. Put $f_{1}=1_{A} f$ and $f_{2}=1_{A^{c}} f$. By the Cauchy-Schwartz inequality we have

$$
\begin{aligned}
\left\|T_{t}\left(f_{1}\right)\right\|_{2}^{2} & =\int_{\mathbf{R}^{d}}\left(\int_{A} u(t, x, y) f_{1}(y) d y\right)^{2} d x \\
& \leq \int_{\mathbf{R}^{d}}\left(\int_{A} u(t, x, y) d y\right)\left(\int_{A} u(t, x, y) f_{1}^{2}(y) d y\right) d x \\
& \leq \int_{A} \int_{\mathbf{R}^{d}} u(t, x, y) f_{1}^{2}(y) d x d y \leq \int_{A} f_{1}^{2}(y) T_{t}\left(1_{\mathbf{R}^{d}}\right)(y) d y \\
& \leq \sup _{y \in A} T_{t}\left(1_{\mathbf{R}^{d}}\right)(y) \int_{A} f_{1}^{2}(y) d y \leq(\varepsilon / 2)^{2}\left\|f_{1}\right\|_{2}^{2} \leq(\varepsilon / 2)^{2} .
\end{aligned}
$$

On the other hand we have

$$
T_{t}\left(f_{2}\right)(x)=\int_{\mathbf{R}^{d}} u(t, x, y) f_{2}(y) d y=\int_{A^{c}} u(t, x, y) f(y) d y=S_{A^{c}}(f)(x) .
$$


Since $N_{\varepsilon / 2}$ is the $(\varepsilon / 2)$-net for $S_{A^{c}}$, there exists $g \in N_{\varepsilon / 2}$ such that $\left\|S_{A^{c}}(f)-g\right\|_{2}<$ $\varepsilon / 2$. Now it is sufficient to show that $\left\|T_{t}(f)-g\right\|_{2}<\varepsilon$. Indeed

$$
\left\|T_{t}(f)-g\right\|_{2}=\left\|T_{t}\left(f_{1}+f_{2}\right)-g\right\|_{2} \leq\left\|S_{A_{c}}(f)-g\right\|_{2}+\left\|T_{t}\left(f_{1}\right)\right\|_{2} \leq \varepsilon .
$$

Thus operator $T_{t}$ is compact.

Now fix $t>0$ and assume that there exists $N>0$ and a sequence of disjoint unit balls $B_{n}=B\left(x_{n}, 1\right)$, such that $V(x)<N$ for any $x \in B_{n}, n \in \mathbb{N}$. We may and do assume there exists $M>2$ such that $\operatorname{dist}\left(B_{n}, B_{m}\right)>M$ for any $n, m \in \mathbb{N}$, $n \neq m$. Note that $M>2$ may be chosen arbitrarily. We will choose appropriate $M>2$ later in the proof. Now consider the sequence $f_{n}=1_{B_{n}} /\left(2\left|B_{n}\right|\right)^{1 / 2}$. All those functions have norms equal to $1 / 2$, and

$$
\begin{aligned}
\left\|T_{t}\left(f_{n}\right)-T_{t}\left(f_{m}\right)\right\|_{2}^{2} & =\frac{1}{2\left|B_{1}\right|} \int_{\mathbf{R}^{d}}\left[\mathbf{E}^{x}\left(X_{t} \in B_{n} ; e^{-\int_{0}^{t} V\left(X_{s}\right) d s}\right)\right. \\
& \left.-\mathbf{E}^{x}\left(X_{t} \in B_{m} ; e^{-\int_{0}^{t} V\left(X_{s}\right) d s}\right)\right]^{2} d x \\
& \geq \frac{1}{2\left|B_{1}\right|} \int_{B\left(x_{n}, 1 / 2\right)}\left[\mathbf{E}^{x}\left(X_{t} \in B_{n} ; e^{-\int_{0}^{t} V\left(X_{s}\right) d s}\right)\right. \\
& \left.-\mathbf{E}^{x}\left(X_{t} \in B_{m} ; e^{-\int_{0}^{t} V\left(X_{s}\right) d s}\right)\right]^{2} d x .
\end{aligned}
$$

To show that the operator $T_{t}$ is not compact, it is sufficient to prove that for $n \neq m$ this norm is greater than a positive constant not depending on $n$ and $m$. To do this we need to estimate both expected values in the last expression. For $x \in B\left(x_{n}, 1 / 2\right)$ we have

$$
\begin{gathered}
\mathbf{E}^{x}\left(X_{t} \in B_{n} ; e^{-\int_{0}^{t} V\left(X_{s}\right) d s}\right) \geq \mathbf{E}^{x}\left(\tau_{B(x, 1 / 2)}>t ; e^{-\int_{0}^{t} V\left(X_{s}\right) d s}\right) \\
\geq e^{-N t} \mathbf{P}^{0}\left(\tau_{B(0,1 / 2)}>t\right)=C_{1}(t) e^{-N t}
\end{gathered}
$$

By Lemma 2.2 for $x \in B\left(x_{n}, 1 / 2\right)$ and $n \neq m$ we have

$$
\begin{aligned}
\mathbf{E}^{x}\left(X_{t} \in B_{m} ; e^{-\int_{0}^{t} V\left(X_{s}\right) d s}\right) & \leq \mathbf{P}^{x}\left(X_{t} \in B_{m}\right) \\
& =\int_{B_{m}} p(t, x, y) d y \leq C_{2} t e^{m t}|M-2|^{-d-\alpha} .
\end{aligned}
$$

Now let us choose $M$ large enough so that $\frac{1}{2} C_{1}(t) e^{-N t}>C_{2} t e^{m t}|M-2|^{-d-\alpha}$. Then we have

$$
\left\|T_{t}\left(f_{n}\right)-T_{t}\left(f_{m}\right)\right\|_{2}^{2} \geq \frac{1}{2\left|B_{1}\right|} \int_{B\left(x_{n}, 1 / 2\right)} \frac{1}{4}\left(C_{1}(t)\right)^{2} e^{-N t} d x .
$$

Thus the points of the sequence $T_{t}\left(f_{n}\right)$ are separated, so the operator $T_{t}$ is not compact.

Now we will show that Condition 1.2 implies IU. Assume that for some open, bounded, nonempty set $D$ and all $t>0$ Condition 1.2 is satisfied. Recall that $\phi_{1}$ is continuous and strictly positive. Therefore there exists $c_{D}>0$ such that for all $x \in D$ we have $\phi_{1}(x) \geq c_{D}$. Hence for any $s>0$ and $x \in \mathbf{R}^{d}$ we have $T_{s}\left(1_{D}\right)(x) \leq$ $T_{s}\left(\phi_{1} / c_{D}\right)(x)=c_{D}^{-1} e^{-\lambda_{1} s} \phi_{1}(x)$. By Lemma 3.1 $u(s, x, y) \leq p(s, x, y) \leq c_{s}, s>0$, 
$x, y \in \mathbf{R}^{d}$. Using the semigroup property we get

$$
\begin{aligned}
& u(t, x, y)=\int_{\mathbf{R}^{d}} \int_{\mathbf{R}^{d}} u(t / 3, x, z) u(t / 3, z, w) u(t / 3, w, y) d z d y \\
& \leq c_{t / 3} \int_{\mathbf{R}^{d}} u(t / 3, x, z) d z \int_{\mathbf{R}^{d}} u(t / 3, w, y) d y=c_{t / 3} T_{t / 3}\left(1_{\mathbf{R}^{d}}\right)(x) T_{t / 3}\left(1_{\mathbf{R}^{d}}\right)(y) \\
& \leq c_{t / 3} c_{t / 3, D}^{2} T_{t / 3}\left(1_{D}\right)(x) T_{t / 3}\left(1_{D}\right)(y) \leq c_{t / 3} c_{t / 3, D}^{2} c_{D}^{-2} e^{-2 \lambda_{1} t / 3} \phi_{1}(x) \phi_{1}(y) .
\end{aligned}
$$

This gives (1.2), so the semigroup $T_{t}$ is IU.

Now we will show that IU implies Condition 1.3. Let $t>0$ and $D$ be an open, bounded, nonempty set. By (1.3) we have

$$
T_{t}\left(1_{D}\right)(x)=\int_{D} u(t, x, y) d y \geq c_{t} \phi_{1}(x) \int_{D} \phi_{1}(y) d y .
$$

Recall that $\phi_{1}$ is bounded. Also by (1.3) we have

$$
T_{t}\left(1_{B(x, 1)}\right)(x) \leq c_{t} \phi_{1}(x) \int_{B(x, 1)} \phi_{1}(y) d y \leq c_{t}|| \phi_{1} \|_{\infty}|B(0,1)| \phi_{1}(x) .
$$

This shows that the inequality (1.3) implies Condition 1.3 ,

\section{Estimates of transition PRobability of the KILLED PROCESS}

In this section we prove some estimates of transition probability of the killed process. These estimates will be used in the next section to estimate the probability of "short jumps". On the other hand it seems that these estimates have not been known before and that they are interesting in themselves. The most general result of such type are Theorem 4.2 and Corollaries 4.4 and 4.5 .

Lemma 4.1. Let $R=B(0,3 / 2) \backslash \overline{B(0,1)}$ and $1<|x|<5 / 4$. Then

$$
\begin{gathered}
\mathbf{E}^{x}\left(\tau_{R}\right) \leq c \delta_{R}^{\alpha / 2}(x), \\
\mathbf{P}^{x}\left(X\left(\tau_{R}\right) \in B^{c}(0,3 / 2)\right) \leq c \delta_{R}^{\alpha / 2}(x) .
\end{gathered}
$$

Here we recall our notation $\delta_{R}(x)=\operatorname{dist}(x, \partial R)$.

Proof. By Theorem 3 in $[\mathrm{R}$, (cf. also [CS3]) we get

$$
G_{R}(x, y) \leq c \min \left\{\frac{\left(\delta_{R}(x) \delta_{R}(y)\right)^{\alpha / 2}}{|x-y|^{d}}, \frac{\delta_{R}^{\alpha / 2}(x)}{|x-y|^{d-\alpha / 2}}\right\} .
$$

Therefore

$$
\mathbf{E}^{x}\left(\tau_{R}\right)=\int_{R} G_{R}(x, y) d y \leq c \int_{R} \frac{\delta_{R}^{\alpha / 2}(x)}{|x-y|^{d-\alpha / 2}} d y \leq c \delta_{R}^{\alpha / 2}(x) .
$$

Let $A=B^{c}(0,3 / 2)$. By Corollary 2.11 and Lemma 2.4 we get

$$
\begin{aligned}
\mathbf{P}^{x}\left(X\left(\tau_{R}\right)\right. & \in A)=\int_{R} G_{R}(x, y) \int_{A} \nu(y-z) d z d y \\
& \leq c \int_{R} G_{R}(x, y) \int_{B^{c}\left(y, \delta_{A}(y)\right)} \frac{1}{|y-z|^{d+\alpha}} d z d y \\
& =c \int_{R} G_{R}(x, y) \int_{\delta_{A}(y)}^{\infty} \frac{r^{d-1}}{r^{d+\alpha}} d r d y=c \int_{R} G_{R}(x, y) \frac{1}{\delta_{A}^{\alpha}(y)} d y .
\end{aligned}
$$


Now we divide the last integral into two parts, over sets $R_{1}=B(0,5 / 4) \backslash \overline{B(0,1)}$ and $R_{2}=B(0,3 / 2) \backslash \overline{B(0,5 / 4)}$. For $y \in R_{1}$ we have $\delta_{A}(y) \geq 1 / 4$, so

$$
\int_{R_{1}} G_{R}(x, y) \frac{1}{\delta_{A}^{\alpha}(y)} d y \leq c \int_{R_{1}} \frac{\delta_{R}^{\alpha / 2}(x)}{|x-y|^{d-\alpha / 2}} 4^{\alpha} d y \leq c \delta_{R}^{\alpha / 2}(x) .
$$

For $y \in R_{2}$ we have $|x-y| \geq 1 / 4$. Hence

$$
\begin{aligned}
\int_{R_{2}} G_{R}(x, y) \frac{1}{\delta_{A}^{\alpha}(y)} d y & \leq c \int_{R_{2}} \frac{\left(\delta_{R}(x) \delta_{R}(y)\right)^{\alpha / 2}}{|x-y|^{d}} \frac{1}{\delta_{A}^{\alpha}(y)} d y \\
& \leq c \int_{R_{2}} \frac{\delta_{R}^{\alpha / 2}(x)}{\delta_{A}^{\alpha / 2}(y)} 8^{d} d y \\
& =c \delta_{R}^{\alpha / 2}(x) \int_{5 / 4}^{3 / 2} \frac{r^{d-1}}{(3 / 2-r)^{\alpha / 2}} d r \leq c \delta_{R}^{\alpha / 2}(x) .
\end{aligned}
$$

The proof of the next theorem contains the main idea of this section.

Theorem 4.2. Let $D=(\overline{B(0,1)})^{c}$ and $T>0$. There exist constants $c_{T}$ and $c$ such that for any $0<t \leq T,|x| \geq 2$ and $1<|y|<5 / 4$ we have

$$
p_{D}(t, x, y) \leq c_{T} e^{-c|x-y|} \delta_{D}^{\alpha / 2}(y) .
$$

Proof. To show this inequality we will estimate the integral of $p_{D}(t, y, z)$ over a small ball $B(x, s)$. Then we will take the limit when $s$ tends to 0 .

Let $R=B(0,3 / 2) \backslash \overline{B(0,1)}$ and $s>0$ such that $B(x, s) \subset B^{c}(0,3 / 2)$. We have

$$
\begin{aligned}
\int_{B(x, s)} p_{D}(t, y, z) d z & =\mathbf{P}^{y}\left(X(t) \in B(x, s), \tau_{D}>t\right) \\
& \leq \mathbf{P}^{y}\left(\tau_{R}<t, X\left(\tau_{R}\right) \in D \backslash R, X(t) \in B(x, s)\right),
\end{aligned}
$$

and by the strong Markov property the last expression is equal to

$$
\mathbf{E}^{y}\left(\tau_{R}<t, X\left(\tau_{R}\right) \in D \backslash R ;\left.\mathbf{P}^{X\left(\tau_{R}\right)}(X(t-r) \in B(x, s))\right|_{r=\tau_{R}}\right) .
$$

Now we divide the set $D \backslash R$ into two subsets, $A=B(x,|x-y| / 16)$ and $F=$ $D \backslash(A \cup R)$. We have $\operatorname{dist}(F, B(x, s)) \geq|x-y| / 32$ for small enough $s$. Note also that $\operatorname{dist}(A, R)=|x|-3 / 2-|x-y| / 16 \geq|x-y| / 16$. The last inequality holds because $|x| \geq|x| / 8+|y| / 8+3 / 2 \geq|x-y| / 8+3 / 2$. Now we are going to estimate the part of (4.3) for the set $F$. At first note that

$$
\begin{aligned}
\mathbf{E}^{y}\left(\tau_{R}<t\right. & \left., X\left(\tau_{R}\right) \in F ;\left.\mathbf{P}^{X\left(\tau_{R}\right)}(X(t-r) \in B(x, s))\right|_{r=\tau_{R}}\right) \\
= & \mathbf{E}^{y}\left(\tau_{R}<t, X\left(\tau_{R}\right) \in F ; \int_{B(x, s)} p\left(t-\tau_{R}, X\left(\tau_{R}\right), z\right) d z\right) .
\end{aligned}
$$


Note also that $X\left(\tau_{R}\right) \in F$, so for $z \in B(x, s)$ we have $\left|X\left(\tau_{R}\right)-z\right| \geq|x-y| / 32 \geq$ $1 / 64$. By Lemma 2.2 this is bounded from above by

$$
\begin{aligned}
& \mathbf{E}^{y}\left(\tau_{R}<t, X\left(\tau_{R}\right) \in F ; \int_{B(x, s)} c t e^{m t} e^{-c^{\prime}\left|z-X\left(\tau_{R}\right)\right|} d z\right) \\
& \quad \leq \mathbf{E}^{y}\left(\tau_{R}<t, X\left(\tau_{R}\right) \in F ;|B(x, s)| c T e^{m T} e^{-c^{\prime}|x-y|}\right) \\
& \quad \leq c_{T}|B(x, s)| e^{-c^{\prime}|x-y|} \mathbf{P}^{y}\left(X\left(\tau_{R}\right) \in D \backslash R\right) .
\end{aligned}
$$

By the previous lemma we finally obtain

$$
\begin{aligned}
\mathbf{E}^{y}\left(\tau_{R}<t, X\left(\tau_{R}\right) \in F ;\right. & \left.\left.\mathbf{P}^{X\left(\tau_{R}\right)}(X(t-r) \in B(x, s))\right|_{r=\tau_{R}}\right) \\
& \leq c_{T}|B(x, s)| e^{-c^{\prime}|x-y|} \delta_{B(0,1)}^{\alpha / 2}(y) .
\end{aligned}
$$

For the set $A$ by the generalized Ikeda-Watanabe formula (Corollary 2.11) we get

$$
\begin{aligned}
& \mathbf{E}^{y}\left(\tau_{R}<t, X\left(\tau_{R}\right) \in A ;\left.\mathbf{P}^{X\left(\tau_{R}\right)}(X(t-r) \in B(x, s))\right|_{r=\tau_{R}}\right) \\
& =\mathbf{E}^{y}\left(\tau_{R}<t, X\left(\tau_{R}\right) \in A ; X(t) \in B(x, s)\right) \\
& =\int_{R} \int_{0}^{t} p_{R}(r, y, z) \int_{A} \nu(z-w) \mathbf{P}^{w}(X(t-r) \in B(x, s)) d w d r d z .
\end{aligned}
$$

Now we will estimate

$$
\int_{A} \nu(z-w) \mathbf{P}^{w}(X(t-r) \in B(x, s)) d w
$$

for $z \in R$ and $r \in(0, t)$. To do this, we need to divide the set $A$ into two subsets $A_{1}=B\left(x, \max \left\{2 s, q^{1 / \alpha} /\left(64 T^{1 / \alpha}\right)\right\}\right)$, where $q=t-r$, and $A_{2}=A \backslash A_{1}$. To make expressions simpler we set $a=\max \left\{2 s, q^{1 / \alpha} /\left(64 T^{1 / \alpha}\right)\right\}$ and $b=|x-y| / 16$. Note that $q^{1 / \alpha} /\left(64 T^{1 / \alpha}\right)<1 / 64<|x-y| / 16$, and we may and do assume that $2 s<|x-y| / 16$. Hence $a<b$. Recall that $\operatorname{dist}(A, R) \geq|x-y| / 16$. For $z \in R$ and $w \in A$ we have $|z-w| \geq|x-y| / 16 \geq 1 / 64$. By Lemma $2.3 \nu(z-w) \leq c e^{-c^{\prime}|z-w|} \leq$ $c e^{-c^{\prime}|x-y|}$.

For the set $A_{2}$ we have

$$
\begin{aligned}
& \int_{A_{2}} \nu(z-w) \mathbf{P}^{w}(X(q) \in B(x, s)) d w \\
& \leq c \int_{A_{2}} e^{-c^{\prime}|x-y|} \int_{B(x, s)} p(q, w, u) d u d w .
\end{aligned}
$$

By Lemma 2.2 this is bounded from above by

$$
\begin{gathered}
c \int_{A_{2}} e^{-c^{\prime}|x-y|} \int_{B(x, s)} \frac{q e^{m q}}{|w-u|^{d+\alpha}} d u d w \leq c \int_{A_{2}} e^{-c^{\prime}|x-y|} \frac{q e^{m T}|B(x, s)|}{\delta_{B(x, s)}^{\alpha+d}(w)} d w \\
\leq c_{T} q e^{-c^{\prime}|x-y|}|B(x, s)| \int_{a}^{b} \frac{r^{d-1}}{(r-s)^{d+\alpha}} d r .
\end{gathered}
$$

Note that $a \geq 2 s$, so this is bounded from above by

$$
c_{T} q e^{-c^{\prime}|x-y|}|B(x, s)| \int_{a}^{\infty} \frac{d r}{r^{1+\alpha}} \leq c_{T} e^{-c^{\prime}|x-y|}|B(x, s)| \frac{q}{a^{\alpha}} .
$$


By the definition of $a$ we have $a \geq q^{1 / \alpha} /\left(64 T^{1 / \alpha}\right)$, hence

$$
\int_{A_{2}} \nu(z-w) \mathbf{P}^{w}(X(q) \in B(x, s)) d w \leq c_{T} e^{-c^{\prime}|x-y|}|B(x, s)| .
$$

We have $A_{1}=B(x, a)$. Recall that for $z \in R$ and $w \in A_{1} \subset A$ we have $\nu(z-w) \leq$ $c e^{-c^{\prime}|x-y|}$. Now we will consider two cases. If $a=2 s$, then $\left|A_{1}\right|=c|B(x, s)|$. Hence

$$
\begin{aligned}
\int_{A_{1}} \nu(z-w) \mathbf{P}^{w}(X(q) \in B(x, s)) d w & \leq \int_{A_{1}} \nu(z-w) d w \\
& \leq c e^{-c^{\prime}|x-y|}|B(x, s)| .
\end{aligned}
$$

If $a=q^{1 / \alpha} /\left(64 T^{1 / \alpha}\right)$, then by Lemma 2.2 we get

$$
\begin{aligned}
\int_{A_{1}} \nu(z-w) & \mathbf{P}^{w}(X(q) \in B(x, s)) d w \\
& \leq c \int_{A_{1}} e^{-c^{\prime}|x-y|} \int_{B(x, s)} p(q, w, u) d u d w \\
& \leq c \int_{A_{1}} e^{-c^{\prime}|x-y|}|B(x, s)| \frac{e^{m q}}{q^{d / \alpha}} d w \\
& \leq c e^{m T} e^{-c^{\prime}|x-y|}|B(x, s)| \frac{\left|A_{1}\right|}{q^{d / \alpha}} .
\end{aligned}
$$

But the Lebesgue measure of $A_{1}$ is equal to $c_{T} q^{d / \alpha}$, so

$$
\int_{A_{1}} \nu(z-w) \mathbf{P}^{w}(X(q) \in B(x, s)) d w \leq c_{T} e^{-c^{\prime}|x-y|}|B(x, s)| .
$$

Substituting (4.7), (4.8) and (4.9) into (4.6) we have

$$
\begin{aligned}
\mathbf{E}^{y}\left(\tau_{R}<t\right. & \left., X\left(\tau_{R}\right) \in A ;\left.\mathbf{P}^{X\left(\tau_{R}\right)}(X(t-r) \in B(x, s))\right|_{r=\tau_{R}}\right) \\
& \leq c_{T} e^{-c^{\prime}|x-y|}|B(x, s)| \int_{R} \int_{0}^{\infty} p_{R}(r, y, z) d r d z \\
& =c_{T} e^{-c^{\prime}|x-y|}|B(x, s)| \int_{R} G_{R}(y, z) d z \\
& =c_{T} e^{-c^{\prime}|x-y|}|B(x, s)| \mathbf{E}^{y}\left(\tau_{R}\right) .
\end{aligned}
$$

By Lemma 4.1 it follows that

$$
\begin{aligned}
\mathbf{E}^{y}\left(\tau_{R}<t, X\left(\tau_{R}\right) \in A\right. & \left.;\left.\mathbf{P}^{X\left(\tau_{R}\right)}(X(t-r) \in B(x, s))\right|_{r=\tau_{R}}\right) \\
& \leq c_{T}|B(x, s)| e^{-c^{\prime}|x-y|} \delta_{B(0,1)}^{\alpha / 2}(y) .
\end{aligned}
$$

From (4.3), (4.5) and (4.10) we finally obtain

$$
\frac{1}{|B(x, s)|} \int_{B(x, s)} p_{D}(t, y, z) d z \leq c_{T} e^{-c^{\prime}|x-y|} \delta_{B(0,1)}^{\alpha / 2}(y) .
$$

Letting $s \longrightarrow 0$ we get the assertion of the theorem.

Corollaries 4.34 .6 below are simple generalizations and conclusions of Theorem 4.2. It seems that estimates of $p_{D}(t, x, y)$ presented in these corollaries have not been known before, and they may find some interesting applications.

Almost the same proof as the proof of Theorem 4.2 leads to a slightly more general fact. 
Corollary 4.3. Let $D=(\overline{B(0, \varepsilon)})^{c}$ and $T>0$. There exist constants $c_{T, \varepsilon}$ and $c_{\varepsilon}$ such that for any $0<t \leq T,|x| \geq 2 \varepsilon$ and $\varepsilon<|y|<5 \varepsilon / 4$ we have

$$
p_{D}(t, x, y) \leq c_{T, \varepsilon} e^{-c_{\varepsilon}|x-y|} \delta_{D}^{\alpha / 2}(y) .
$$

Corollary 4.4. Let $T>0$ and let $D \subset \mathbf{R}^{d}$ be an open set satysfying the outer ball condition in point $z \in \partial D$, that is, there exists a ball $B(w, \varepsilon) \subset D^{c}, \varepsilon>0$, such that $\partial D \cap \partial B(w, \varepsilon)=\{z\}$. Then there exist constants $c_{T, \varepsilon}$ and $c_{\varepsilon}$ such that for any $0<t \leq T, x, y \in D$ and $|x-w|>2 \varepsilon$ we have

$$
p_{D}(t, x, y) \leq \frac{c_{T, \varepsilon}}{|x-y|^{d+\alpha}} e^{-c_{\varepsilon}|x-y|}|z-y|^{\alpha / 2} .
$$

Proof. We will consider two cases. First let $|z-y|>\varepsilon / 4$. Then the assertion follows from Lemma 2.2. Now assume that $|z-y|<\varepsilon / 4$. Then $|y-w|<5 \varepsilon / 4$. $B(w, \varepsilon)$ is a subset of $D^{c}$, thus $p_{D}(t, x, y) \leq p_{(\overline{B(w, \varepsilon)})^{c}}(t, x, y)$ for any $x, y \in \mathbf{R}^{d}$, and any $t>0$. By the previous corollary

$$
p_{(\overline{B(w, \varepsilon)})^{c}}(t, x, y) \leq c_{T, \varepsilon} e^{-c_{\varepsilon}|x-y|} \delta_{B(w, \varepsilon)}^{\alpha / 2}(y),
$$

for any $x \notin B(w, 2 \varepsilon)$. Besides $\delta_{B(w, \varepsilon)}(y) \leq|z-y|$ and $|x-y| \geq 3 \varepsilon / 4$, thus

$$
p_{D}(t, x, y) \leq p_{(\overline{B(w, \varepsilon)})^{c}}(t, x, y) \leq \frac{c_{T, \varepsilon}}{|x-y|^{d+\alpha}} e^{-c_{\varepsilon}|x-y|}|z-y|^{\alpha / 2} .
$$

Corollary 4.5. Let $T>0$ and let $D$ be a set with a uniform outer ball condition, i.e. there exists $\varepsilon>0$, such that for any $z \in \partial D$ there exists $B(w, \varepsilon) \subset D^{c}$, such that $\partial B(w, \varepsilon) \cap \partial D=\{z\}$. Then there exist constants $c_{T, \varepsilon}$ and $c_{\varepsilon}$ such that for any $x, y \in D, \delta_{D}(x)>\varepsilon$ and $0<t \leq T$ we have

$$
p_{D}(t, x, y) \leq \frac{c_{T, \varepsilon}}{|x-y|^{d+\alpha}} e^{-c_{\varepsilon}|x-y|} \delta_{D}^{\alpha / 2}(y) .
$$

Corollary 4.6. Let $R \geq 1, D=(\overline{B(0, R)})^{c}$ and $0<T<\infty$. There exist constants $c_{T}$ and $c$ such that for any $0<t \leq T,|x| \geq R+1$ and $R<|y| \leq R+1 / 4$ we have

$$
p_{D}(t, x, y) \leq c_{T} e^{-c|x-y|} \delta_{D}^{\alpha / 2}(y) .
$$

As an application of the above corollary we prove the following proposition, which will be very important in the proof of the main theorem.

Proposition 4.7. Let $R \geq 1, D=(\overline{B(0, R)})^{c}$ and $0<T<\infty$. There exists a constant $c_{T}$ such that for any $0 \leq t_{1}<t_{2} \leq T$ and $|x|>R+1$ we have

$$
\mathbf{P}^{x}\left(t_{1}<\tau_{D}<t_{2}\right) \leq c_{T}\left(t_{2}-t_{1}\right) .
$$

Let us emphasize that $c_{T}$ does not depend on $R$.

Proof. By the generalized Ikeda-Watanabe formula (Corollary 2.11)

$$
\mathbf{P}^{x}\left(t_{1}<\tau_{D}<t_{2}\right)=\int_{D} \int_{t_{1}}^{t_{2}} p_{D}(s, x, y) d s \int_{D^{c}} \nu(y-z) d z d y .
$$

Now we divide the set $D$ into two parts, $A=B^{c}(0, R+1 / 4)$ and $B=D \backslash A$. If $y \in A$ and $z \in D^{c}$, then $|z-y| \geq 1 / 4$. Hence by Lemma $2.3 \nu(y-z) \leq c e^{-c^{\prime}|y-z|}$. 
It follows that

$$
\begin{aligned}
\int_{A} \int_{t_{1}}^{t_{2}} p_{D}(s, x, y) d s & \int_{D^{c}} \nu(y-z) d z d y \\
& \leq c \int_{A} \int_{t_{1}}^{t_{2}} p_{D}(s, x, y) d s \int_{D^{c}} e^{-c^{\prime}|y-z|} d z d y .
\end{aligned}
$$

We have $\int_{D^{c}} e^{-c^{\prime}|y-z|} d z \leq \int_{\mathbf{R}^{d}} e^{-c^{\prime}|y-z|} d z<\infty$. Therefore (4.11) is bounded from above by

$$
c \int_{t_{1}}^{t_{2}} \int_{A} p_{D}(s, x, y) d y d s=c \int_{t_{1}}^{t_{2}} \mathbf{P}^{x}\left(\tau_{D}>s\right) d s \leq c\left(t_{2}-t_{1}\right) .
$$

Let $\delta(y)=\delta_{B(0, R)}(y)$. By Lemma 2.4 we have

$$
\begin{aligned}
\int_{B} \int_{t_{1}}^{t_{2}} p_{D}(s, x, y) d s & \int_{D^{c}} \nu(y-z) d z d y \\
& \leq c \int_{B} \int_{t_{1}}^{t_{2}} p_{D}(s, x, y) d s \int_{B^{c}(y, \delta(y))} \frac{1}{|y-z|^{d+\alpha}} d z d y \\
& =c \int_{B} \int_{t_{1}}^{t_{2}} p_{D}(s, x, y) d s \int_{\delta(y)}^{\infty} \frac{r^{d-1}}{r^{d+\alpha}} d r d y \\
& =c \int_{B} \int_{t_{1}}^{t_{2}} p_{D}(s, x, y) d s \frac{1}{\delta^{\alpha}(y)} d y
\end{aligned}
$$

and by Corollary 4.6 this is bounded from above by

$$
c_{T}\left(t_{2}-t_{1}\right) \int_{B} e^{-c|x-y|} \frac{d y}{\delta^{\alpha / 2}(y)} .
$$

It is sufficient to show that this integral is bounded by a constant not depending on $R$. We will prove this for $d \geq 3$. For the dimension $d=1$ the proof is very easy and for $d=2$ the proof is similar to the proof for $d \geq 3$.

We may and do assume that $x=(0, \ldots, 0, R+1)$. We introduce spherical coordinates in $\mathbf{R}^{d}\left(r, \varphi_{1}, \varphi_{2}, \ldots, \varphi_{d-1}\right), r \in[0, \infty), \varphi_{1}, \ldots, \varphi_{d-2} \in[0, \pi], \varphi_{d-1} \in$ $[0,2 \pi)$, with center in 0 and principal axis $0 x$.

We divide the set $B$ into 2 parts:

$$
\begin{aligned}
& B_{1}=\left\{\left(r, \varphi_{1}, \varphi_{2}, \ldots, \varphi_{d-1}\right): r \in(R, R+1 / 4), \varphi_{1} \in[0,1 / R]\right\}, \\
& B_{2}=\left\{\left(r, \varphi_{1}, \varphi_{2}, \ldots, \varphi_{d-1}\right): r \in(R, R+1 / 4), \varphi_{1} \in(1 / R, \pi]\right\} .
\end{aligned}
$$

We have

$$
\begin{aligned}
\int_{B_{1}} e^{-c|x-y|} \frac{d y}{\delta^{\alpha / 2}(y)} & \leq c \int_{0}^{1 / R} \int_{R}^{R+1 / 4} \frac{r^{d-1}}{(r-R)^{\alpha / 2}} \sin ^{d-2} \varphi_{1} d r d \varphi_{1} \\
& \leq c R^{d-1} \int_{0}^{1 / R} \int_{0}^{1 / 4} \frac{1}{r^{\alpha / 2}} \varphi_{1}^{d-2} d r d \varphi_{1} \\
& \leq c R^{d-1}(1 / R)^{d-1}=c .
\end{aligned}
$$

Now we estimate the integral over $B_{2}$. Let $y \in B_{2}$ and put $y_{0}=y R /|y|$ and $x_{0}=x R /|x|=(0,0, \ldots, R)$. Note that $|x-y| \geq\left|x_{0}-y\right|$ and $\left|x_{0}-y\right| \geq\left|x_{0}-y_{0}\right|-1 / 4$. Let $y=\left(r, \varphi_{1}, \ldots, \varphi_{d-1}\right)$ in spherical coordinates. Since $y \in B_{2}$ we have $\varphi_{1}>1 / R$. 
It follows that $\left|x_{0}-y_{0}\right| \geq 2 R \sin (1 / 2 R) \geq 2 / \pi>1 / 2$. Hence $\left|x_{0}-y\right| \geq\left|x_{0}-y_{0}\right| / 2$. This gives

$$
|x-y| \geq\left|x_{0}-y_{0}\right| / 2=R \sin \left(\varphi_{1} / 2\right) \geq R \varphi_{1} / \pi=C R \varphi_{1} .
$$

We also have $e^{-c|x-y|} \leq c^{\prime}|x-y|^{-d}$. Therefore $e^{-c|x-y|} \leq c^{\prime}\left(C R \varphi_{1}\right)^{-d}$. It follows that

$$
\begin{aligned}
\int_{B_{2}} e^{-c|x-y|} \frac{d y}{\delta^{\alpha / 2}(y)} & \leq c^{\prime \prime} \int_{1 / R}^{\pi} \int_{R}^{R+1 / 4} \frac{r^{d-1}}{(r-R)^{\alpha / 2}} \frac{\sin ^{d-2} \varphi_{1}}{\left(R \varphi_{1}\right)^{d}} d r d \varphi_{1} \\
& \leq c R^{d-1} \int_{0}^{1 / 4} \frac{1}{r^{\alpha / 2}} d r \int_{1 / R}^{\pi} \frac{\varphi_{1}^{d-2}}{\left(R \varphi_{1}\right)^{d}} d \varphi_{1} \\
& =c R^{-1} \int_{1 / R}^{\pi} \varphi_{1}^{-2} d \varphi_{1}=c .
\end{aligned}
$$

\section{INTRINSIC ULTRACONTRACTIVITY}

In this section we prove the main result of this paper, Theorem 1.6, In this section we fix the potential $V \in \mathcal{V}$.

To prove that the semigroup $T_{t}$ is IU we will use Condition 1.2 for $D=B(0,1)$. To show that the semigroup $T_{t}$ is not IU we will use Condition 1.3 . At first we prove two auxiliary lemmas, which will be used to estimate $T_{t}\left(1_{D}\right)(x)$.

Lemma 5.1. Let $0 \leq t_{1}<t_{2} \leq t<\infty, x \in \mathbf{R}^{d},|x| \geq 3, D=B(0,1)$ and $B=B(x, 1)$. Then

$$
\mathbf{P}^{x}\left(X\left(\tau_{B}\right) \in D / 2, t_{1}<\tau_{B}<t_{2}\right) \geq \frac{c_{t}\left(t_{2}-t_{1}\right)}{|x|^{\frac{d+\alpha+1}{2}}} e^{-m^{1 / \alpha}|x|} .
$$

Proof. By Proposition 2.5 we have

$$
\begin{aligned}
\mathbf{P}^{x}\left(X\left(\tau_{B}\right)\right. & \left.\in D / 2, t_{1}<\tau_{B}<t_{2}\right) \\
& =\int_{B} \int_{t_{1}}^{t_{2}} p_{B}(s, x, y) d s \int_{D / 2} \nu(y-z) d z d y .
\end{aligned}
$$

By Lemma 2.3 this is bounded from below by

$$
\begin{aligned}
\int_{B} \int_{t_{1}}^{t_{2}} & p_{B}(s, x, y) d s|D / 2| \frac{c}{(|x|+1)^{\frac{d+\alpha+1}{2}}} e^{-m^{1 / \alpha}(|x|+1)} d y \\
& \geq \frac{c}{|x|^{\frac{d+\alpha+1}{2}}} e^{-m^{1 / \alpha}|x|} \int_{t_{1}}^{t_{2}} \int_{B} p_{B}(s, x, y) d y d s \\
& =\frac{c}{|x|^{\frac{d+\alpha+1}{2}}} e^{-m^{1 / \alpha}|x|} \int_{t_{1}}^{t_{2}} \mathbf{P}^{x}\left(\tau_{B}>s\right) d s \\
& \geq \frac{c\left(t_{2}-t_{1}\right)}{|x|^{\frac{d+\alpha+1}{2}}} e^{-m^{1 / \alpha}|x|} \mathbf{P}^{x}\left(\tau_{B}>t\right)
\end{aligned}
$$

and $P^{x}\left(\tau_{B}>t\right)=c_{t}$. Here we recall our convention that constants $c$ may change their value from one use to the next. 
Lemma 5.2. For any $r \geq 0$ we have

$$
\sum_{n=1}^{\infty} \frac{e^{-r / n}}{n(n+1)} \geq \frac{e^{-1}}{r+1}
$$

Proof. We have

$$
\sum_{n=1}^{\infty} \frac{e^{-r / n}}{n(n+1)} \geq \sum_{n=[r]+1}^{\infty} \frac{e^{-1}}{n(n+1)}=\frac{e^{-1}}{[r]+1} \geq \frac{e^{-1}}{r+1} .
$$

Now we will show some lemmas needed to estimate $T_{t}\left(1_{\mathbf{R}^{d}}\right)(x)$ from above. This is the most difficult part of the paper. Roughly speaking, the main idea is to divide $\mathbf{R}^{d}$ into appropriate rings, and estimate the probability of jumps between these rings. To shorten notation, from now on we will assume that $n, k, l, N \in \mathbb{N}$. We will use the following notation:

- $2 \leq n_{0} \in N$ will be chosen later,

- $R_{n}=\overline{B(0, n)} \backslash \overline{B(0, n-1)}$ for any $n \geq n_{0}+2$,

- $R_{n_{0}}=B\left(0, n_{0}\right), R_{n_{0}+1}=B\left(0, n_{0}+1\right)$,

- $A_{n}=(\overline{B(0, n-2)})^{c}$ for any $n \geq n_{0}+2$,

- $A_{n_{0}}=\mathbf{R}^{d}, A_{n_{0}+1}=\mathbf{R}^{d}$,

- $\tau_{n}=\tau_{A_{n}}=\inf \left\{t \geq 0: X_{t} \notin A_{n}\right\}, n \geq n_{0}$,

- $\sigma_{n}=\sigma_{R_{n}}=\inf \left\{t \geq 0: X_{t} \in R_{n}\right\}, n \geq n_{0}$.

Let us point out that for $n \geq n_{0}+2$ sets $R_{n}$ are "rings" in $\mathbf{R}^{d}$ and $R_{n_{0}}, R_{n_{0}+1}$ are balls. Note also that $\tau_{n_{0}}=\infty, \tau_{n_{0}+1}=\infty$.

First we need to estimate a volume of the intersection of two balls.

Lemma 5.3. Let $0<l \leq n, k>0$. Consider two balls $B(x, n)$ and $B(y, k+l)$ with $|x-y|=n+k$. The volume of the intersection of these balls (denoted by I) is less than $\mathrm{cl}^{\frac{d+1}{2}}(\min \{2 k+l, 2 n\})^{\frac{d-1}{2}}$.

Proof. Let $d>1$. Let $z$ be any point belonging to the intersection of spheres with centers at $x$ and $y$, and radius $n$ and $k+l$. Let $w$ be the orthogonal projection of $z$ on the line containing $x$ and $y$. Then $k<|w-y|<k+l$. Therefore

$$
|z-w|^{2}=(k+l)^{2}-|w-y|^{2}<(k+l)^{2}-k^{2}=2 k l+l^{2}=l(2 k+l) .
$$

Analogical argument can be applied to $n$, thus we have

$$
|z-w|^{2}<n^{2}-(n-l)^{2}=2 n l-l^{2}<2 n l .
$$

Therefore we get

$$
|I| \leq c l|z-w|^{d-1} \leq c l^{\frac{d+1}{2}}(\min \{2 k+l, 2 n\})^{\frac{d-1}{2}} .
$$

Let $d=1$. In this case the lemma obviously holds, because the intersection of two balls (intervals) has measure equal to $l$.

Now we estimate an integral of the Levy measure.

Lemma 5.4. Let $|x| \geq N \geq n+1$ and $n \geq n_{0}$. Then we have

$$
\int_{R_{n}} \nu(x-y) d y \leq \frac{c(\min \{N-n, n\})^{\frac{d-1}{2}}}{(N-n)^{\frac{d+\alpha+1}{2}}} e^{-m^{1 / \alpha}(N-n)} .
$$


Proof. By Lemma 2.3

$$
\begin{aligned}
\int_{R_{n}} \nu(x-y) d y & \leq \int_{R_{n}} \frac{c}{|x-y|^{\frac{d+\alpha+1}{2}}} e^{-m^{1 / \alpha}|x-y|} d y \\
& \leq \int_{B(0, n)} \frac{c}{|x-y|^{\frac{d+\alpha+1}{2}}} e^{-m^{1 / \alpha}|x-y|} d y=: J
\end{aligned}
$$

Let us point out that the last inequality holds both when $n \geq n_{0}+2$ (and $R_{n}$ are "rings") and when $n=n_{0}$ or $n=n_{0}+1$ (and $R_{n}$ are balls).

It is sufficient to consider only the case $x=(0,0, \ldots, 0, N)$. Let $N-n=k$. Consider a sequence of balls $B(x, k+l)$ for $1 \leq l \leq n$. Then

$$
\begin{aligned}
B(0, n) & =\underbrace{(B(0, n) \backslash B(x, k+n))}_{=A} \\
& \cup \bigcup_{0<l \leq n}^{\{(B(0, n) \cap B(x, k+l)) \backslash B(x, k+l-1)\}} .
\end{aligned}
$$

In addition we denote $E_{l}=B(0, n) \cap B(x, k+l)$. We will divide an integral $J$ into integrals over sets $A$ and $A_{l}$. At first we estimate an integral over $A$ :

$$
\begin{aligned}
\int_{A} \frac{c}{|x-y|^{\frac{d+\alpha+1}{2}}} e^{-m^{1 / \alpha}|x-y|} d y & \leq \frac{c|A|}{(k+n)^{\frac{d+\alpha+1}{2}}} e^{-m^{1 / \alpha}(k+n)} \\
& \leq \frac{c n^{d}}{k^{\frac{d+\alpha+1}{2}}} e^{-m^{1 / \alpha}(k+n)} \leq \frac{c}{k^{\frac{d+\alpha+1}{2}}} e^{-m^{1 / \alpha} k}
\end{aligned}
$$

In the last inequality we use the fact that there exists $c$ (depending on $m, \alpha$ and $d$ ) such that for any $n \in \mathbb{N}$ we have $n^{d} e^{-m^{1 / \alpha} n} \leq c$. In the sequel we will use similar inequalities without further comments. For $A_{l}$ we have

$$
\begin{aligned}
\int_{A_{l}} \frac{c}{|x-y|^{\frac{d+\alpha+1}{2}}} e^{-m^{1 / \alpha}|x-y|} d y & \leq \frac{c\left|A_{l}\right|}{(k+l-1)^{\frac{d+\alpha+1}{2}}} e^{-m^{1 / \alpha}(k+l-1)} \\
& \leq \frac{c\left|E_{l}\right|}{k^{\frac{d+\alpha+1}{2}}} e^{-m^{1 / \alpha}(k+l)}
\end{aligned}
$$

Now we will consider two cases. At first assume that $k \geq n$. Using the previous lemma we obtain

$$
\begin{aligned}
J & \leq \frac{c}{k^{\frac{d+\alpha+1}{2}}} e^{-m^{1 / \alpha} k}+\sum_{1 \leq l \leq n} \frac{c l^{\frac{d+1}{2}}(\min \{2 k+l, 2 n\})^{\frac{d-1}{2}}}{k^{\frac{d+\alpha+1}{2}}} e^{-m^{1 / \alpha}(k+l)} \\
& \leq \frac{c}{k^{\frac{d+\alpha+1}{2}}} e^{-m^{1 / \alpha} k}+\frac{c(\min \{3 k, 3 n\})^{\frac{d-1}{2}}}{k^{\frac{d+\alpha+1}{2}}} e^{-m^{1 / \alpha} k} \sum_{1 \leq l \leq n} l^{\frac{d+1}{2}} e^{-m^{1 / \alpha} l} \\
& \leq \frac{c(\min \{k, n\})^{\frac{d-1}{2}}}{k^{\frac{d+\alpha+1}{2}}} e^{-m^{1 / \alpha} k} .
\end{aligned}
$$


In the second case $(k<n)$ we have

$$
\begin{aligned}
J & \leq \frac{c}{k^{\frac{d+\alpha+1}{2}}} e^{-m^{1 / \alpha} k}+\sum_{1 \leq l \leq n} \frac{c l^{\frac{d+1}{2}}(\min \{2 k+l, 2 n\})^{\frac{d-1}{2}}}{k^{\frac{d+\alpha+1}{2}}} e^{-m^{1 / \alpha}(k+l)} \\
& \leq \frac{c}{k^{\frac{d+\alpha+1}{2}}} e^{-m^{1 / \alpha} k}+\sum_{1 \leq l \leq k} \frac{c l^{\frac{d+1}{2}}(\min \{3 k, 3 n\})^{\frac{d-1}{2}}}{k^{\frac{d+\alpha+1}{2}}} e^{-m^{1 / \alpha}(k+l)} \\
& +\sum_{k<l \leq n} \frac{c l^{\frac{d+1}{2}}(3 l)^{\frac{d-1}{2}}}{k^{\frac{d+\alpha+1}{2}}} e^{-m^{1 / \alpha}(k+l)} \leq \frac{c(\min \{k, n\})^{\frac{d-1}{2}}}{k^{\frac{d+\alpha+1}{2}}} e^{-m^{1 / \alpha} k} .
\end{aligned}
$$

Lemma 5.5. For $N-1<|x| \leq N, n_{0} \leq n \leq N-2$ and $0 \leq t_{1}<t_{2} \leq T<\infty$ we have

$$
\begin{aligned}
& \mathbf{P}^{x}\left(X\left(\tau_{N}\right) \in R_{n}, t_{1}<\tau_{N}<t_{2}\right) \\
& \leq C_{T}\left(t_{2}-t_{1}\right) \frac{(\min \{N-n, n\})^{\frac{d-1}{2}}}{(N-n)^{\frac{d+\alpha+1}{2}}} e^{-m^{1 / \alpha}(N-n)} .
\end{aligned}
$$

Proof. Recall that $\tau_{N}=\tau_{A_{N}}$. For $n=N-2$ we have

$$
\mathbf{P}^{x}\left(X\left(\tau_{N}\right) \in R_{n}, t_{1}<\tau_{N}<t_{2}\right) \leq \mathbf{P}^{x}\left(t_{1}<\tau_{N}<t_{2}\right) \leq c_{T}\left(t_{2}-t_{1}\right),
$$

by Proposition 4.7 .

Let $n<N-2$. By Proposition 2.7 (the generalization of the Ikeda-Watanabe formula) we have

$$
\begin{aligned}
\mathbf{P}^{x}\left(X\left(\tau_{N}\right) \in R_{n},\right. & \left.t_{1}<\tau_{N}<t_{2}\right) \\
= & \int_{A_{N}} \int_{t_{1}}^{t_{2}} p_{A_{N}}(s, x, y) d s \int_{R_{n}} \nu(y-z) d z d y .
\end{aligned}
$$

Note that for $y \in A_{N}$ we have $|y|>N-2$. By Lemma 5.4 for $y \in A_{N}$ we have

$$
\int_{R_{n}} \nu(y-z) d z \leq \frac{c \min \{N-2-n, n\}^{\frac{d-1}{2}}}{(N-2-n)^{\frac{d+\alpha+1}{2}}} e^{-m^{1 / \alpha}(N-2-n)} .
$$

Therefore (5.1) is bounded from the above by

$$
\frac{c(\min \{N-n, n\})^{\frac{d-1}{2}}}{(N-n)^{\frac{d+\alpha+1}{2}}} e^{-m^{1 / \alpha}(N-n)} \int_{t_{1}}^{t_{2}} \int_{A_{N}} p_{A_{N}}(s, x, y) d y d s .
$$

We have $\int_{A_{N}} p_{A_{N}}(s, x, y) d y=\mathbf{P}^{x}\left(\tau_{A_{N}}>s\right) \leq 1$, and the lemma follows.

Lemma 5.6. For any $r>0$ we have

$$
\sum_{n=1}^{\infty} \frac{e^{-\frac{r}{n+1}}}{n(n+1)} \leq \frac{5}{r}
$$

Proof. We have

$$
\sum_{n=1}^{\infty} \frac{e^{-\frac{r}{n+1}}}{n(n+1)}=\frac{e^{-r / 2}}{2}+\sum_{n=2}^{\infty} \frac{e^{-\frac{r}{n+1}}}{n(n+1)}
$$

Note that $1 /\left(2 e^{r / 2}\right) \leq 1 / r$. We also have

$$
\sum_{n=2}^{\infty} \frac{e^{-\frac{r}{n+1}}}{n(n+1)} \leq \int_{2}^{\infty} \frac{e^{-\frac{r}{s+1}}}{(s-1) s} d s \leq 2 \int_{2}^{\infty} \frac{e^{-\frac{r}{2 s}}}{s^{2}} d s=\frac{4}{r} \int_{0}^{r / 4} e^{-u} d u \leq \frac{4}{r} .
$$


Lemma 5.7. For $N-1<|x| \leq N, n_{0} \leq k \leq N-2, t>0$ and $a>0$ we have

$$
\begin{aligned}
& \mathbf{E}^{x}\left(\tau_{N}<t, X\left(\tau_{N}\right) \in R_{k} ; e^{-\tau_{N} a}\right) \\
& \leq \mathcal{C}_{1}(t) \frac{(\min \{N-k, k\})^{\frac{d-1}{2}}}{(N-k)^{\frac{d+\alpha+1}{2}}} e^{-m^{1 / \alpha}(N-k)} \frac{1}{a} .
\end{aligned}
$$

Proof. Using last two lemmas we obtain

$$
\begin{aligned}
\mathbf{E}^{x}\left(\tau_{N}<t\right. & \left.X\left(\tau_{N}\right) \in R_{k} ; e^{-\tau_{N} a}\right) \\
& =\sum_{i=1}^{\infty} \mathbf{E}^{x}\left(\frac{t}{i+1} \leq \tau_{N}<\frac{t}{i}, X\left(\tau_{N}\right) \in R_{k} ; e^{-\tau_{N} a}\right) \\
& \leq \sum_{i=1}^{\infty} e^{\frac{-t a}{i+1}} \mathbf{P}^{x}\left(\frac{t}{i+1} \leq \tau_{N}<\frac{t}{i}, X\left(\tau_{N}\right) \in R_{k}\right) \\
& =\sum_{i=1}^{\infty} e^{\frac{-t a}{i+1}} C_{t} \frac{t}{i(i+1)} \frac{(\min \{N-k, k\})^{\frac{d-1}{2}}}{(N-k)^{\frac{d+\alpha+1}{2}}} e^{-m^{1 / \alpha}(N-k)} \\
& \leq \mathcal{C}_{1}(t) \frac{(\min \{N-k, k\})^{\frac{d-1}{2}}}{(N-k)^{\frac{d+\alpha+1}{2}}} e^{-m^{1 / \alpha}(N-k)} \frac{1}{a} .
\end{aligned}
$$

Of course the constant $C_{1}(t)$ also depends on $\alpha, d$ and $m$.

Now we define events, sequences of jumps, which will help to estimate $T_{t}\left(1_{\mathbf{R}^{d}}\right)$. This notation is very essential in the sequel. Roughly speaking the main idea of the paper is to estimate $T_{t}\left(1_{\mathbf{R}^{d}}\right)$ by estimating the appropriate jumps of the process between rings. This idea comes from the paper [BK].

For $k \geq n_{0}, n \geq k+2$ and $t>0$ we define

- $S(n, k, 1, t)=\left\{X\left(\tau_{n}\right) \in R_{k}, \sigma_{k}<t\right\}$,

- $S(n, k, l, t)=\bigcup_{p=k+2}^{n-2} S(n, p, l-1, t) \cap S(p, k, 1, t)$ for any $l \geq 2$,

- $R(n, k, l, t)=S(n, k, l, t) \cap\left\{\tau_{k}>t\right\}$.

When $n \geq k+2, n-2<k+2$ and $l \geq 2$, one should understand that $S(n, k, l, t)=$ $\emptyset$ and $R(n, k, l, t)=\emptyset$. Note also that for fixed $n$ and $k$ the events $S(n, k, l, t)$ are empty for large enough $l$.

$S(n, k, 1, t)$ is the event that the process while leaving $A_{n}$ jumps directly to $R_{k}$ and $\sigma_{k}=\inf \left\{t \geq 0: X_{t} \in R_{k}\right\}$, the entry time to $R_{k}$, is smaller than $t$. Note that if $X\left(\tau_{n}\right) \in R_{k}$, then $\sigma_{k}=\tau_{n}$.

$S(n, p, 1, t) \cap S(p, k, 1, t)$ for $n-2 \geq p \geq k+2$ is the event that the process while leaving $A_{n}$ jumps directly to $R_{p}$ and then while leaving $A_{p}$ jumps directly to $R_{k}$ and $\sigma_{k}<t, \sigma_{p}<t$. $S(n, k, 2, t)$ for $n-2 \geq k+2(l=2)$ is the sum of $S(n, p, 1, t) \cap S(p, k, 1, t)$ for all $p$ between $k+2$ and $n-2$. Roughly one may think of $S(n, k, 2, t)$ as the event that the process goes from $A_{n}$ to $R_{k}$ in 2 "appropriate jumps" (and $\sigma_{k}<t$ ).

Similarly, by induction we define $S(n, k, l, t)$. One may think of $S(n, k, l, t)$ as the event that the process goes from $A_{n}$ to $R_{k}$ in $l$ "appropriate jumps" (and $\sigma_{k}<t$ ).

$R(n, k, l, t)$ is the event that the process goes from $A_{n}$ to $R_{k}$ in $l$ "appropriate jumps", $\sigma_{k}<t$, and that the process remains in $A_{k}$ from $\sigma_{k}$ to $t$.

Note that "jumps" are defined so that when the process jumps to $R_{p}=\overline{B(0, p)} \backslash$ $B(0, p-1)$, then the next jump is from $A_{p}=(\overline{B(0, p-2)})^{c}\left(\operatorname{not}\right.$ from $\left.(\overline{B(0, p-1)})^{c}\right)$. 
This is done for technical reasons. It would be difficult to estimate the probability of jumps from $R_{p}$ to $(\overline{B(0, p-1)})^{c}$. The same method was used in $[\mathrm{BK}$.

Let $t>0, n \geq 2 n_{0}+4$ and $x \in R_{n}$. We take $n \geq 2 n_{0}+4$ so that $[n / 2] \geq n_{0}+2$. Note also that $n-[n / 2] \geq 2$. For such $t, n$ and $x$ we have

$$
\begin{array}{r}
T_{t}\left(1_{\mathbf{R}^{d}}\right)(x)=\mathbf{E}^{x}\left(e^{-\int_{0}^{t} V\left(X_{s}\right) d s}\right) \leq \mathbf{E}^{x}\left(\tau_{[n / 2]}>t ; e^{-\int_{0}^{t} V\left(X_{s}\right) d s}\right) \\
+\sum_{k=n_{0}+2}^{[n / 2]} \sum_{l=1}^{\infty} \mathbf{E}^{x}\left(R(n, k, l, t) ; e^{-\int_{0}^{t} V\left(X_{s}\right) d s}\right) \\
+\sum_{k=n_{0}}^{n_{0}+1} \sum_{l=1}^{\infty} \mathbf{E}^{x}\left(R(n, k, l, t) ; e^{-\int_{0}^{t} V\left(X_{s}\right) d s}\right) .
\end{array}
$$

The terms under the sum $\sum_{k=n_{0}+2}^{[n / 2]} \sum_{l=1}^{\infty}$ correspond to events that the process will make $l$ "appropriate jumps" from $A_{n}$ to $R_{k}$ and then remain in $A_{k}$ up to time $t$. The terms under the sum $\sum_{k=n_{0}}^{n_{0}+1} \sum_{l=1}^{\infty}$ correspond to events that the process will make $l$ "appropriate jumps" from $A_{n}$ to $R_{k}$ and here we do not control the behaviour of the process after $\sigma_{k}\left(\tau_{n_{0}}=\infty, \tau_{n_{0}+1}=\infty\right)$. Roughly speaking these terms for $k=n_{0}$ and $k=n_{0}+1$ appear separately because we can control the expression of the type $\mathbf{E}^{x}\left(S(n, k, l, t), e^{-\frac{1}{2} \int_{0}^{\sigma_{k}} V\left(X_{s}\right) d s}\right)$ only when $k$ is big enough (see Lemma 5.9). We have 2 parameters $k=n_{0}$ and $k=n_{0}+1$ for technical reasons (because of our definition of jumps).

Now we focus on estimating the single terms from the above sums. For $x \in R_{n}$, $k \geq n_{0}+2, n \geq k+2, l \geq 1$ and $t>0$ we have

$$
\begin{aligned}
\mathbf{E}^{x}\left(R(n, k, l, t) ; e^{-\int_{0}^{t} V\left(X_{s}\right) d s}\right) \\
\leq \mathbf{E}^{x}\left(R(n, k, l, t) ; e^{-\frac{1}{2} \int_{0}^{\sigma_{k}} V\left(X_{s}\right) d s} e^{-\frac{1}{2} \int_{0}^{t} V\left(X_{s}\right) d s}\right) \\
\leq \mathbf{E}^{x}\left(S(n, k, l, t), \tau_{k}>t ; e^{-\frac{1}{2} \int_{0}^{\sigma_{k}} V\left(X_{s}\right) d s} e^{-\frac{t}{2} L(k-2)}\right) \\
\leq e^{-\frac{t}{2} L(k-2)} \mathbf{E}^{x}\left(S(n, k, l, t) ; e^{-\frac{1}{2} \int_{0}^{\sigma_{k}} V\left(X_{s}\right) d s}\right) .
\end{aligned}
$$

By similar arguments we get for $k=n_{0}$ and $k=n_{0}+1$

$$
\mathbf{E}^{x}\left(R(n, k, l, t) ; e^{-\int_{0}^{t} V\left(X_{s}\right) d s}\right) \leq \mathbf{E}^{x}\left(S(n, k, l, t) ; e^{-\frac{1}{2} \int_{0}^{\sigma_{k}} V\left(X_{s}\right) d s}\right) .
$$

Now we need the following auxiliary fact.

Lemma 5.8. Let $n>k>l>0$. We have

$$
W=\frac{\min \{n-k, k\}}{n-k} \frac{\min \{k-l, l\}}{k-l} \leq \mathcal{C}_{2} \frac{\min \{n-l, l\}}{n-l} .
$$

Proof. One can easily see that the following inequality holds:

$$
(n-k)(k-l) \geq \frac{1}{2}(n-l) \min \{n-k, k-l\} .
$$

Thus

Hence it is sufficient to show that

$$
W \leq 2 \frac{\min \{n-k, k\} \min \{k-l, l\}}{\min \{n-k, k-l\}} \frac{1}{(n-l)} .
$$

$$
Y=\frac{\min \{n-k, k\} \min \{k-l, l\}}{\min \{n-k, k-l\}} \leq c \min \{n-l, l\} .
$$


Now we have three cases:

- $n-k \leq k-l$,

$$
Y=\min \{k-l, l\} \leq \min \{n-l, l\},
$$

- $n-k>k-l$ and $k-l \leq l$,

$$
Y=\min \{n-k, k\} \leq \min \{n-l, 2 l\} \leq 2 \min \{n-l, l\},
$$

- $n-k>k-l$ and $k-l>l$,

$$
Y=\frac{\min \{n-k, k\} l}{k-l} \leq \frac{k l}{k / 2}=2 l
$$

and

$$
Y=\frac{\min \{n-k, k\} l}{k-l} \leq \frac{(n-k)(k-l)}{k-l}=n-k \leq n-l .
$$

The following lemma is crucial in our considerations. Roughly speaking we estimate $e^{-\int_{0}^{t} V\left(X_{s}\right) d s}$ depending on the number of "appropriate jumps" the process made. The idea of the proof of this lemma is taken from [BK], Lemma 4.5.

In this lemma we will not use our convention that constants may change their value from line to line. All constants which appear in the formulation and the proof of the lemma will not change their value. This is because in the induction proof we need to know that constants do not depend on the parameter $l$.

Lemma 5.9. Fix $t>0$. Let $\mathcal{C}_{3}(t)=16 \tilde{c}^{2} \mathcal{C}_{1}(t)$, where $\tilde{c}$ is a constant from Definition 1.4 and $\mathcal{C}_{1}(t)$ is a constant from Lemma [5.7. Let $n_{0} \in \mathbb{N}\left(n_{0}\right.$ depends on $\left.t\right)$ be large enough so that

$$
L\left(n_{0}\right) \geq 1 \quad \text { and } \quad \frac{\mathcal{C}_{3}(t)}{L\left(n_{0}\right)} 8 \mathcal{C}_{2}{ }^{\frac{d-1}{2}} \sum_{p=1}^{\infty} \frac{1}{p^{1+\frac{\alpha}{2}}} \leq 1,
$$

where $\mathcal{C}_{2}$ is a constant from Lemma 5.8 .

Then for $x \in R_{n}, k \geq n_{0}, n \geq k+2, l \geq 1$ we have

$$
\mathbf{E}^{x}\left(S(n, k, l, t) ; e^{-\frac{1}{2} \int_{0}^{\sigma_{k}} V\left(X_{s}\right) d s}\right) \leq \frac{\mathcal{C}_{3}(t)}{2^{l}} e^{-m^{1 / \alpha}(n-k)} \frac{(\min \{n-k, k\})^{\frac{d-1}{2}}}{(n-k)^{\frac{d+\alpha+1}{2}} L(n)} .
$$

Recall that (Definition 1.4) the function $L(n)$ is nondecreasing and tending to $\infty$, so for large enough $n_{0}$ (5.6) holds. We also point out that in the proof of Theorem 1.6 there will be some additional conditions on $n_{0}$.

Proof. First let $l=1$. This is the case when the process makes only one appropriate "jump". Recall that $S(n, k, 1, t)=\left\{X\left(\tau_{n}\right) \in R_{k}, \sigma_{k}<t\right\}$ and for $X\left(\tau_{n}\right) \in R_{k}$ we have $\tau_{n}=\sigma_{k}$. Note that for $s \in\left[0, \tau_{n}\right)$ we have $X_{s} \in A_{n}=(\overline{B(0, n-2)})^{c}$, so by Definition $1.5 V\left(X_{s}\right) \geq L(n-2)$. Using Lemma 5.7 we obtain

$$
\begin{gathered}
\mathbf{E}^{x}\left(S(n, k, 1, t) ; e^{-\frac{1}{2} \int_{0}^{\sigma_{k}} V\left(X_{s}\right) d s}\right) \leq \mathbf{E}^{x}\left(X\left(\tau_{n}\right) \in R_{k}, \tau_{n}<t ; e^{-\tau_{n} L(n-2) / 2}\right) \\
\leq \mathcal{C}_{1}(t) e^{-m^{1 / \alpha}(n-k)} \frac{(\min \{n-k, k\})^{\frac{d-1}{2}}}{(n-k)^{\frac{d+\alpha+1}{2}}} \frac{2}{L(n-2)} .
\end{gathered}
$$

Recall that $n \geq k+2 \geq n_{0}+2$, so $L(n-1), L(n-2)$ are no smaller than $L\left(n_{0}\right) \geq 1$. By Definition $1.4(3) L(n) \leq \tilde{c} L(n-1)+\tilde{c} \leq 2 \tilde{c} L(n-1)$. Similarly $L(n) \leq 4 \tilde{c}^{2} L(n-2)$. 
Therefore for $\mathcal{C}_{3}(t)=16 \tilde{c}^{2} \mathcal{C}_{1}(t)$ (5.7) holds for $l=1$. We will show that for such $\mathcal{C}_{3}(t)$ (5.7) also holds for all $l \geq 1$. We will prove (5.7) by induction on $l$. Recall once again that in this proof we will not use our convention that the constants may change their value from one use to the next.

Let $l \geq 2$. Suppose we have proved (5.7) for $1, \ldots, l-1$ and all $k \geq n_{0}, n \geq k+2$. We will show (5.7) for $l$. By Definition of $S(n, k, l, t)$ we have

$$
\begin{aligned}
& \mathbf{E}^{x}\left(S(n, k, l, t) ; e^{-\frac{1}{2} \int_{0}^{\sigma_{k}} V\left(X_{s}\right) d s}\right) \\
& =\sum_{p=k+2}^{n-2} \mathbf{E}^{x}(\underbrace{S(n, p, l-1, t)}_{=A}, S(p, k, 1, t) ; e^{-\frac{1}{2} \int_{0}^{\sigma_{p}} V\left(X_{s}\right) d s} e^{-\frac{1}{2} \int_{\sigma_{p}}^{\sigma_{k}} V\left(X_{s}\right) d s}) \\
& \leq \sum_{p=k+2}^{n-2} \mathbf{E}^{x}\left(A, S\left(p, k, 1, t+\sigma_{p}\right) ; e^{-\frac{1}{2} \int_{0}^{\sigma_{p}} V\left(X_{s}\right) d s} e^{-\frac{1}{2} \int_{\sigma_{p}}^{\sigma_{k}} V\left(X_{s}\right) d s}\right) .
\end{aligned}
$$

By the strong Markov property the last expression is equal to

$$
\sum_{p=k+2}^{n-2} \mathbf{E}^{x}\left(A ; e^{-\frac{1}{2} \int_{0}^{\sigma p} V\left(X_{s}\right) d s} \mathbf{E}^{X\left(\sigma_{p}\right)}\left(S(p, k, 1, t) ; e^{-\frac{1}{2} \int_{0}^{\sigma_{k}} V\left(X_{s}\right) d s}\right)\right) .
$$

Now by our induction hypothesis this is bounded from above by

$$
\begin{gathered}
\sum_{p=k+2}^{n-2} \frac{\mathcal{C}_{3}(t)}{2^{l-1}} e^{-m^{1 / \alpha}(n-p)} \frac{(\min \{n-p, p\})^{\frac{d-1}{2}}}{(n-p)^{\frac{d+\alpha+1}{2}}} \frac{1}{L(n)} \\
\times \frac{\mathcal{C}_{3}(t)}{2} e^{-m^{1 / \alpha}(p-k)} \frac{(\min \{p-k, k\})^{\frac{d-1}{2}}}{(p-k)^{\frac{d+\alpha+1}{2}}} \frac{1}{L(p)} .
\end{gathered}
$$

The function $L(n)$ is nondecreasing, so this is bounded from above by

$$
\frac{\mathcal{C}_{3}(t)}{2^{l}} \frac{e^{-m^{1 / \alpha}(n-k)}}{L(n)} \frac{\mathcal{C}_{3}(t)}{L\left(n_{0}\right)} \sum_{p=k+2}^{n-2} \frac{(\min \{n-p, p\})^{\frac{d-1}{2}}}{(n-p)^{\frac{d+\alpha+1}{2}}} \frac{(\min \{p-k, k\})^{\frac{d-1}{2}}}{(p-k)^{\frac{d+\alpha+1}{2}}} .
$$

By Lemma 5.8 and (5.5) the last sum is smaller than

$$
\begin{aligned}
& \sum_{p=k+2}^{n-2} \mathcal{C}_{2} \frac{d-1}{2} \frac{(\min \{n-k, k\})^{\frac{d-1}{2}}}{(n-k)^{\frac{d+\alpha+1}{2}}} \frac{2^{1+\alpha / 2}}{(\min \{n-p, p-k\})^{1+\frac{\alpha}{2}}} \\
& \leq 4 \mathcal{C}_{2} \frac{d-1}{2} \frac{(\min \{n-k, k\})^{\frac{d-1}{2}}}{(n-k)^{\frac{d+\alpha+1}{2}}} \sum_{p=k+2}^{n-2}\left(\frac{1}{(n-p)^{1+\frac{\alpha}{2}}}+\frac{1}{(p-k)^{1+\frac{\alpha}{2}}}\right) \\
& \leq 8 \mathcal{C}_{2}{ }^{\frac{d-1}{2}} \frac{(\min \{n-k, k\})^{\frac{d-1}{2}}}{(n-k)^{\frac{d+\alpha+1}{2}}} \sum_{p=1}^{\infty} \frac{1}{p^{1+\frac{\alpha}{2}}}
\end{aligned}
$$

Eventually, by (5.8) and (5.6) we obtain

$$
\mathbf{E}^{x}\left(S(n, k, l, t) ; e^{-\frac{1}{2} \int_{0}^{\sigma} k} V\left(X_{s}\right) d s\right) \leq \frac{\mathcal{C}_{3}(t)}{2^{l}} e^{-m^{1 / \alpha}(n-k)} \frac{(\min \{n-k, k\})^{\frac{d-1}{2}}}{(n-k)^{\frac{d+\alpha+1}{2}} L(n)} .
$$

Now we can prove the main result of this paper. 
Proof of Theorem 1.6. To prove intrinsic ultracontractivity of $T_{t}$ we will use Condition 1.2, that is, for all $t>0$ there exists $c_{t}$ such that for all $x \in \mathbf{R}^{d}, T_{t}\left(1_{\mathbf{R}^{d}}\right)(x) \leq$ $c_{t} T_{t}\left(1_{D}\right)(x)$, where $D=B(0,1)$.

First we estimate $T_{t}\left(1_{\mathbf{R}^{d}}\right)(x)$. Fix $t>0$. We assume that $n_{0}$ satisfies condtion (5.6) from Lemma 5.9. Let $n \geq 2 n_{0}+4$ and $x \in R_{n}$. Applying the last lemma and (5.4) to the equality (5.3), we have

$$
\begin{aligned}
T_{t}\left(1_{\mathbf{R}^{d}}\right)(x) & \leq \mathbf{E}^{x}\left(\tau_{[n / 2]}>t ; e^{-\int_{0}^{t} V\left(X_{s}\right) d s}\right) \\
& +\sum_{k=n_{0}+2}^{[n / 2]} \sum_{l=1}^{\infty} \frac{\mathcal{C}_{3}(t)}{L(n)} \frac{1}{2^{l}} e^{-m^{1 / \alpha}(n-k)} e^{-\frac{t}{2} L(k-2)} \frac{k^{\frac{d-1}{2}}}{(n-k)^{\frac{d+\alpha+1}{2}}} \\
& +\sum_{k=n_{0}}^{n_{0}+1} \sum_{l=1}^{\infty} \frac{\mathcal{C}_{3}(t)}{L(n)} \frac{1}{2^{l}} e^{-m^{1 / \alpha}(n-k)} \frac{k^{\frac{d-1}{2}}}{(n-k)^{\frac{d+\alpha+1}{2}}}=I+I I+I I I .
\end{aligned}
$$

We have

$$
I \leq e^{-t L([n / 2]-2)} .
$$

Note that the function $L$ is comparable on unit intervals (see Definition 1.4). Therefore there exists a constant $A>0$ such that $L(n) \leq e^{A n}$. We also have $L(n) / n \rightarrow \infty$. Let us recall that $t>0$ is fixed. Thus we can choose $n_{0}$ (depending on $t$ ) large enough so that for $n \geq n_{0}$ we have $t L([n / 2]-2)>m^{1 / \alpha} n+(A+1) n$. For such $n_{0}$ we have

$$
I \leq e^{-t L([n / 2]-2)} \leq e^{-m^{1 / \alpha} n} e^{-(A+1) n} \leq \frac{c}{L(n)} e^{-m^{1 / \alpha} n} \frac{1}{n^{\frac{d+\alpha+1}{2}}} .
$$

By similar arguments we can choose $n_{0}$ large enough so that for $k \geq n_{0}$ we have $t L(k-2) / 2-m^{1 / \alpha} k>k$. Note also that $k \leq[n / 2]$, so we have $n-k \geq n / 2$. For such $n_{0}$ we have

$$
I I \leq \frac{c_{t}}{L(n)} \frac{e^{-m^{1 / \alpha} n}}{n^{\frac{d+\alpha+1}{2}}} \sum_{k=n_{0}+2}^{[n / 2]} e^{-k} k^{\frac{d-1}{2}} \leq \frac{c_{t}}{L(n)} e^{-m^{1 / \alpha} n} \frac{1}{n^{\frac{d+\alpha+1}{2}}} .
$$

For $I I I$ we get similar estimates. It follows that for $n_{0}$ chosen as above, $x \in R_{n}$ and $n \geq 2 n_{0}+4$ we have

$$
T_{t}\left(1_{\mathbf{R}^{d}}\right)(x) \leq I+I I+I I I \leq \frac{c_{t}}{L(n)} e^{-m^{1 / \alpha} n} \frac{1}{n^{\frac{d+\alpha+1}{2}}} .
$$

Recall that by (5.6) we have $L\left(n_{0}\right) \geq 1$. By Definition 1.5 for $|x| \geq n_{0}$ we get that $V(x) \leq C L(|x|)+C \leq 2 C L(|x|)$, so $L(|x|)$ and $V(x)$ are comparable. Since $n \geq|x|>n-1\left(x \in R_{n}\right)$ we get

$$
T_{t}\left(1_{\mathbf{R}^{d}}\right)(x) \leq \frac{c_{t}}{V(x)+1} e^{-m^{1 / \alpha}|x|} \frac{1}{(|x|+1)^{\frac{d+\alpha+1}{2}}},
$$

for $|x| \geq 2 n_{0}+4$.

Now we estimate $T_{t}\left(1_{D}\right)(x)$. Fix $t>0$. Recall that $D=B(0,1)$ and let $|x| \geq$ $n_{0}+1, B=B(x, 1)$ (recall that $n_{0} \geq 2$ so $|x| \geq 3$ ). We have

$$
\begin{aligned}
& T_{t}\left(1_{D}\right)(x)=\mathbf{E}^{x}\left(X_{t} \in D ; e^{-\int_{0}^{t} V\left(X_{s}\right) d s}\right) \\
& \geq \mathbf{E}^{x}\left(X\left(\tau_{B}\right) \in D / 2, \tau_{B}<t, \forall_{s \in\left[\tau_{B}, t+\tau_{B}\right]} X_{s} \in D ; e^{-\int_{0}^{\tau_{B}} V\left(X_{s}\right) d s} e^{-\int_{\tau_{B}}^{t+\tau_{B}} V\left(X_{s}\right) d s}\right) \\
& \geq c_{t} \mathbf{E}^{x}\left(X\left(\tau_{B}\right) \in D / 2, \tau_{B}<t, \forall_{s \in\left[\tau_{B}, t+\tau_{B}\right]} X_{s} \in D ; e^{-\int_{0}^{\tau_{B}} V\left(X_{s}\right) d s}\right) .
\end{aligned}
$$


By the strong Markov property this is equal to

$$
\begin{gathered}
c_{t} \mathbf{E}^{x}\left(X\left(\tau_{B}\right) \in D / 2, \tau_{B}<t ; e^{-\int_{0}^{\tau_{B}} V\left(X_{s}\right) d s} \mathbf{P}^{X\left(\tau_{B}\right)}\left(\tau_{D}>t\right)\right) \\
\geq c_{t} \mathbf{E}^{x}\left(X\left(\tau_{B}\right) \in D / 2, \tau_{B}<t ; e^{-\int_{0}^{\tau_{B}} V\left(X_{s}\right) d s}\right) .
\end{gathered}
$$

For $y \in B$ we have $|y|<|x|+1$, so for $s \in\left[0, \tau_{B}\right)$ we get $\left|X_{s}\right|<|x|+1$. Since $|x| \geq n_{0}+1$ we have $L(|x|+1) \geq 1$. Hence for $s \in\left[0, \tau_{B}\right)$ we have $V\left(X_{s}\right) \leq$ $C L(|x|+1)+C \leq 2 C L(|x|+1) \leq c^{\prime} L(|x|)$. It follows that

$$
\begin{aligned}
T_{t}\left(1_{D}\right)(x) & \geq c_{t} \sum_{i=1}^{\infty} \mathbf{E}^{x}\left(X\left(\tau_{B}\right) \in D / 2, \frac{t}{i+1} \leq \tau_{B}<\frac{t}{i} ; e^{-\int_{0}^{\tau_{B}} V\left(X_{s}\right) d s}\right) \\
& \geq c_{t} \sum_{i=1}^{\infty} e^{-t c^{\prime} L(|x|) / i} \mathbf{P}^{x}\left(X\left(\tau_{B}\right) \in D / 2, \frac{t}{i+1}<\tau_{B}<\frac{t}{i}\right) .
\end{aligned}
$$

By Lemmas 5.1 and 5.2 we get

$$
\begin{aligned}
T_{t}\left(1_{D}\right)(x) & \geq c_{t} \sum_{i=1}^{\infty} e^{-t c^{\prime} L(|x|) / i} \frac{t}{i(i+1)} \frac{1}{|x|^{\frac{d+\alpha+1}{2}}} e^{-m^{1 / \alpha}|x|} \\
& \geq \frac{c_{t}}{|x|^{\frac{d+\alpha+1}{2}}} e^{-m^{1 / \alpha}|x|} \frac{1}{t c^{\prime} L(|x|)+1} \\
& \geq \frac{c_{t}}{(|x|+1)^{\frac{d+\alpha+1}{2}}(V(x)+1)} e^{-m^{1 / \alpha}|x|} .
\end{aligned}
$$

This and (5.9) gives Condition 1.2 for $|x| \geq 2 n_{0}+4$. For small $x$ this condition is obvious, because both sides are bounded, continuous and bounded away from 0 . Therefore the semigroup $T_{t}$ is IU.

When $T_{t}$ is IU we can also show asymptotic behavior of the first eigenfunction $\phi_{1}(x)$ :

$$
\phi_{1}(x)=e^{-\lambda_{1}} T_{1}\left(\phi_{1}\right)(x) \leq e^{-\lambda_{1}}\left(\sup _{x \in \mathbf{R}^{d}} \phi_{1}(x)\right) T_{1}\left(1_{\mathbf{R}^{d}}\right)(x)=C T_{1}\left(1_{\mathbf{R}^{d}}\right)(x),
$$

because $\phi_{1}(x)$ is bounded.

On the other hand for $D=B(0,1)$ we have

$$
\begin{aligned}
\phi_{1}(x) & =e^{-\lambda_{1}} T_{1}\left(\phi_{1}\right)(x) \geq e^{-\lambda_{1}} T_{1}\left(1_{D} \phi_{1}\right)(x) \\
& \geq e^{-\lambda_{1}}\left(\inf _{x \in D} \phi_{1}(x)\right) T_{1}\left(1_{D}\right)(x)=c T_{1}\left(1_{D}\right)(x),
\end{aligned}
$$

because $\phi_{1}(x)$ is strictly positive, and continuous. Now (5.9) and (5.10) give (1.5) for large $x\left(|x| \geq 2 n_{0}+4\right)$. For small $x$ (1.5) is trivial because $\phi_{1}$ is continuous and strictly positive.

Finally we will show that if $V \in \mathcal{V}$ and condition $\lim _{|x| \rightarrow \infty} V(x) /|x|=\infty$ is not satisfied, then the semigroup $T_{t}$ is not intrinsically ultracontractive. Since $V \in \mathcal{V}$ we have $\lim _{|x| \rightarrow \infty} V(x)=\infty$. So there exists $r_{0}>1$ such that for $|x|>r_{0}$ we have $V(x)>1$. For such $x$ we get $L(|x|) \leq V(x) \leq 2 C L(|x|)$. $L$ is comparable on unit intervals, so $V$ is comparable on unit balls. Therefore we may assume that there exists a constant $M<\infty$ and a sequence of balls $B_{n}=B\left(x_{n}, 1\right),\left|x_{n}\right| \rightarrow \infty$, $\left|x_{n}\right|>r_{0}+1,\left|x_{n}\right| \geq 3, n \geq 1$, such that $V(x)>1, V(x) /|x|<M$ for any $x \in \bigcup_{n=1}^{\infty} B_{n}$.

Now we will show that Condition 1.3 does not hold. This implies that the semigroup $T_{t}$ is not IU. 
Let $D=B(0,1)$ and $x \in \bigcup_{n=1}^{\infty} B_{n}$. We have

$$
T_{t}\left(1_{D}\right)(x)=\mathbf{E}^{x}\left(X_{t} \in D ; e^{-\int_{0}^{t} V\left(X_{s}\right) d s}\right) \leq \mathbf{P}^{x}\left(X_{t} \in D\right)=\int_{D} p(t, x, y) d y .
$$

Since $\left|x_{n}\right| \geq 3$ we have $|x| \geq 2$ and $|x-y| \geq 1$ for $y \in D$. By Lemma 2.2 we get

$$
T_{t}\left(1_{D}\right)(x) \leq c_{1} t e^{m t}|D| e^{-c_{2}(|x|-1)} .
$$

To estimate $T_{t}\left(1_{\mathbf{R}^{d}}\right)(x)$ we will again use the fact that $V$ is comparable on unit balls. That is, there exists a constant $c_{3}$ such that for $x \in \bigcup_{n=1}^{\infty} B_{n}$ we have $\sup _{y \in B(x, 1)} V(y) \leq c_{3} V(x)$. It follows that

$$
\begin{aligned}
T_{t}\left(1_{B(x, 1)}\right)(x) & \geq \mathbf{E}^{x}\left(\tau_{B(x, 1)}>t ; e^{-\int_{0}^{t} V\left(X_{s}\right) d s}\right) \\
& \geq \mathbf{P}^{x}\left(\tau_{B(x, 1)}>t\right) e^{-t c_{3} V(x)} \geq \mathbf{P}^{0}\left(\tau_{B(0,1)}>t\right) e^{-t c_{3} M|x|} \\
& =c_{t} e^{-t c_{3} M|x|} .
\end{aligned}
$$

If we choose $t$ small enough to have $c_{2}>t c_{3} M$, then Condition 1.3 will not be satisfied for large enough $x$. This implies that the semigroup $T_{t}$ is not IU.

\section{REFERENCES}

[B] R. Bañuelos, Intrinsic ultracontractivity and eigenfunction estimates for Schrödinger operators, J. Funct. Anal. 100 (1991), 181-206. MR.1124298 (92k:35066)

[BD] R. Bañuelos and B. Davis, A geometrical characterization of intrinsic ultracontractivity for planar domains with boundaries given by the graphs of functions, Indiana Univ. Math. J. 41 (1992), no. 4, 885-913. MR1206335 (94g:60142)

[Ba1] D. Bakry, Étude des transformations de Riesz dans les variétés Riemanniennes à courbure négative minoirée, Séminaire de Probabilités XXI, Lect. Notes in Math. 1247 (1987), 137172. MR0941980(89h:58208)

[Ba2] D. Bakry, La propriété de sous-harmonicité des diffusions dans les variétés, Séminaire de Probabilités XXII, Lect. Notes in Math. 1321 (1988), 1-50. MR.0960507 (90h:58084)

[Bo] K. Bogdan, The boundary Harnack principle for the fractional Laplacian, Studia Math. 123 (1997), no. 1, 43-80. MR.1438304 (98g:31005)

[BB1] K. Bogdan and T. Byczkowski, Potential theory for the $\alpha$-stable Schrödinger operator on bounded Lipschitz domains, Studia Math. 133(1) (1999), 53-92. MR1671973 (99m:31010)

[BB2] K. Bogdan and T. Byczkowski, Potential theory of Schrödinger operator based on fractional Laplacian, Prob. Math. Statist. 20 (2000), 293-335. MR1825645 (2002a:31002)

[BK] K. Burdzy and T. Kulczycki, Stable processes have thorns, Ann. Probab. 31 (2003), no. 1, 170-194. MR1959790 (2003k:60079)

[C1] R. Carmona, Path integrals for relativistic Schrödinger operators, Lect. Notes in Phys. 345 (1989), 65-92. MR1037317 (91g:60088)

[CMS] R. Carmona, W.C. Masters and B. Simon, Relativistic Schrödinger operators: asymptotic behavior of the eigenfunctions, J. Funct. Anal. 91 (1990), 117-142. MR1054115|(91i:35139)

[CS1] Z. Chen, R. Song, Intrinsic ultracontractivity and conditional gauge for symmetric stable processes, J. Funct. Anal. 150 (1997), no. 1, 204-239. MR1473631 (98j:60103)

[CS2] Z. Chen, R. Song, Intrinsic ultracontractivity, conditional lifetimes and conditional gauge for symmetric stable processes on rough domains, Illinois J. Math. 44 (2000), no. 1, 138160. MR1731385 (2001m:60166)

[CS3] Z. Chen, R. Song, Estimates of Green functions and Poisson kernels for symmetric stable processes, Math. Ann. 312 (1998), 465-501. MR1654824 (2000b:60179)

[CZ] K.L. Chung, Z. Zhao "From Brownian Motion to Schrödinger's equation", Springer, New York, 1995. MR:1329992 (96f:60140)

[Db] I. Daubechies, One electron molecules with relativistic kinetic energy: properties of discrete spectrum, Comm. Math. Phys. 94 (1984), 523-535. MR0763750(86j:81023)

[DL] I. Daubechies and E.H. Lieb, One electron relativistic molecules with Coulomb interaction, Comm. Math. Phys. 90 (1983), 297-310. MR0719430 (85j:81007) 
[D] E. B. Davies, "Heat Kernels and Spectral Theory", Cambridge Univ. Press, Cambridge, 1989. MR0990239 (90e:35123)

[DS] E. B. Davies and B. Simon, Ultracontractivity and heat kernels for Schrödinger operators and Dirichlet Laplacians, J. Funct. Anal. 59 (1984), 335-395. MR0766493 (86e:47054)

[Da] B. Davis, Intrinsic ultracontractivity and the Dirichlet Laplacian, J. Funct. Anal. 100 (1991), no. 1, 162-180. MR.1124297 (92k:35065)

[F] C. Fefferman, The $N$-body problem in quantum machanics, Comm. Pure and Appl. Math. 39 (1986), S67-S109. MR0861484 (88e:81171a)

[FL] C. Fefferman and R. de la Llave, Relativistic stability of matter-I, Rev. Math. Iberoamericana 2 (1986), 119-223. MR0864658 (88g:81155)

[H] I.W. Herbst, Spectral theory of the operator $\left(p^{2}+m^{2}\right)^{1 / 2}-Z e^{2} / r$, Comm. Math. Phys. 53 (1977), 285-294. MR0436854 (55:9790)

[IW] N. Ikeda and S. Watanabe, On some relations between the harmonic measure and the Levy measure for a certain class of Markov processes, J. Math. Kyoto Univ. 2 (1962), 79-95. MR0142153(25:5546)

[K] T. Kulczycki, Intrinsic ultracontractivity for symmetric stable processes, Bull. Polish Acad. Sci. Math. 46 (1998), 325-334. MR.1643611(99j:60115)

[L] E. Lieb, The stability of matter, Rev. Modern Phys. 48 (1976), 553-569. MR0456083 $(56: 14314)$

[LY] E. Lieb and H.T. Yau, Stability and instability of relativistic matter, Comm. Math. Phys. 118 (1988), no. 2, 177-213. MR0956165 (90c:81251)

[R] M. Ryznar, Estimates of Green function for relativistic $\alpha$-stable process, Potential Analysis 17 (2002), 1-23. MR 1906405 (2003f:60087)

[S] B. Simon, Schrödinger semigroups, Bull. Amer. Math. Soc. 7 (1982), no. 3, 447-526. MR0670130(86b:81001a)

[Z] Z. Zhao, A probabilistic principle and generalized Schrödinger perturbation, J. Funct. Anal. 101 (1991), no. 1, 162-176. MR:1132313 (93f:60116)

Institute of Mathematics, WrocŁaw University of Technology, Wyb. Wyspianskiego 27, 50-370 WrocŁaw, Poland

E-mail address: tkulczyc@im.pwr.wroc.pl

Department of Mathematics, Purdue University, West Lafayette, Indiana 47906

E-mail address: siudeja@math.purdue.edu 